\title{
MINIMAL FUNCTIONAL BASES FOR ELASTICITY TENSOR SYMMETRY CLASSES
}

\author{
R. DESMORAT, N. AUFFRAY, B. DESMORAT, M. OLIVE, AND B. KOLEV
}

\begin{abstract}
Functional bases, synonymous with separating sets, are usually formulated for an entire vector space, such as the space $\mathbb{E}$ la of elasticity tensors. We propose here to define functional bases limited to symmetry strata, i.e., sets of tensors of the same symmetry class. We provide such lowcardinality minimal bases for tetragonal, trigonal, cubic or transversely isotropic symmetry strata of the elasticity tensor.
\end{abstract}

\section{INTRODUCTION}

In the field of linear elasticity, the mechanical properties of an elastic material are represented by an elasticity tensor $\mathbf{E}$, element of the vector space $\mathbb{E}$ la. This association is nevertheless not unique since two elasticity tensors, that differ only up to a rotation, describe the same elastic material [17]. It is important, for applications, to be able to distinguish within $\mathbb{E}$ la which tensors represent the same materials from those who do not. The answer to this question is provided by the construction of a finite set $\mathscr{F}$ preferably minimal - of $\mathrm{SO}(3)$-invariant functions (simply called invariant functions in the following), which

(1) enable one to check if two elasticity tensors describe the same elastic material, i.e., that they are related by a rotation;

(2) allow one to rewrite any invariant function $f$ of an elasticity tensor $\mathbf{E}$ as a function of the elements of $\mathscr{F}$ (i.e., rewrite $f(\mathbf{E})=F(\mathscr{F})$ for some function $F$ ).

This second point constitutes the core of the application of Invariant Theory to Continuum Mechanics [35, $39,6,40,38]$.

The knowledge of an integrity basis provides an answer to this twofold question, but, generally, the cardinality of a minimal integrity basis can be very high. For instance, in the case of three-dimensional elasticity, a minimal integrity basis consists of 294 elements [27, 29]. This is mainly due to the fact that an integrity basis is a response to a different mathematical question, namely, the determination of a set of generators for the algebra of $\mathrm{SO}(3)$-invariant polynomial functions over $\mathbb{E l a}^{1}$.

An invariant set which satisfies (1) is called a separating set, while one which satisfies (2) is called a functional basis [42]. Although they seem different at first glance, these two notions are in fact equivalent, as shown by Wineman and Pipkin [43]. This is interesting since the cardinality of a functional basis can be lower than the one of an integrity basis. But, in contrast to integrity bases and despite some attempts $[16,25]$, there is no general algorithm to obtain functional bases.

For isotropic elasticity, it is well-known that Lamé parameters $\lambda, \mu$ are two invariants that allow to separate isotropic elasticity tensors and to write invariant functions of an isotropic elasticity tensor $\mathbf{E}$ (any invariant function $f(\mathbf{E})$ can be written as $f(\mathbf{E})=F(\lambda, \mu)$ for some function $F$ ). The extension of this simple observation to the whole vector space $\mathbb{E}$ la is a difficult problem, as emphasized by Ming et al [23]. Indeed, these authors have obtained a polynomial functional basis of 251 elements, still a rather large number! There are in the literature different strategies to reduce the number of elements of a functional basis. For instance,

- change the class of its elements: usually polynomial invariants are considered [35, 44, 26, 29, 13, $22,24]$, but this is not mandatory;

- look for local separating sets instead of global ones: the separating property is then defined, not on the whole vector space, but only on a neighbourhood of a given tensor. In this direction, Bona

Date: January 19, 2022.

2010 Mathematics Subject Classification. 74B05; 74E10 ; 15A72.

Key words and phrases. Anisotropy; Covariants; Invariant theory; Symmetry classes.

Three of the authors, R. Desmorat, B. Kolev and M. Olive, were partially supported by CNRS Projet 80-Prime GAMM (Géométrie algébrique complexe/réelle et mécanique des matériaux).

${ }^{1}$ Any invariant polynomial in the components $E_{i j k l}$ of $\mathbf{E}$ can be written as a polynomial in the elements of the integrity basis of the elasticity tensors. 
et al. [8] proposed a local parametrization of orbits of generic triclinic elasticity tensors by 18 local algebraic invariants. A separating set of 18 local polynomial invariants was provided in [15, Theorem A.3];

- restrict the separating property to a subset of generic tensors (generally triclinic). The corresponding functional bases are then called weak functional bases [7].

When combined, these strategies lead to a drastic reduction in the cardinality of a functional basis. For three-dimensional elasticity tensors, a weak separating set of 39 global polynomial invariants has been provided in [7], and a weak separating set of 18 global rational invariants has been obtained in [15, Corollary 4.5]. Nevertheless, to reduce this set from 294 elements to only 18, a price has to be paid, some (in general non triclinic) elasticity tensors are a priori excluded from the possibility to check them.

The approach followed here is complementary. Instead of considering the whole vector space $\mathbb{E}$ la, we are looking for sets of invariants which separate tensors of a given symmetry class, with no genericity restrictions. Our aim is then to produce optimal functional bases, on these lower-dimensional elasticity symmetry classes of $\mathbb{E}$ la. In this paper, we will achieve this task for trigonal, tetragonal, transverse isotropic, and cubic elasticity tensors. Our work strongly relies on the geometry of fourth-order harmonic tensors [3] and elasticity tensors [29].

Outline. The eight symmetry classes of linear elasticity and the associated breaking symmetry diagram (due to [17]) are recalled in section 2, where we summarize necessary and sufficient polynomial conditions (obtained in [29]) for an elasticity tensor to belong to a given symmetry stratum (i.e., a set of elasticity tensors of the same symmetry class). In section 3, we introduce the mathematical material necessary to define rigorously the notion of minimal functional bases, not only on the whole elasticity tensor space $\mathbb{E}$ la but also - and this is the originality of the present work - on each symmetry stratum. We illustrate this method, first in section 4, by the construction of minimal functional bases for the orthotropic and the transversely isotropic strata of the space of second-order symmetric tensors, and, then, in section 5 , by one for the orthotropic, the tetragonal, the trigonal and the transversely isotropic strata of the space of fourthorder harmonic tensors (which appear in the harmonic decomposition of elasticity tensors). Thanks to the key-definition of a non vanishing second-order covariant, we obtain, in an intrinsic manner, our main result in section 6 and section 7 , which is the explicit formulation of low-cardinality functional bases for elasticity tensors at least tetragonal or trigonal.

Tensorial operations. Using the Euclidean structure of $\mathbb{R}^{3}$, no distinction will be made between covariant, contravariant or mixed tensors. All tensor components will be expressed with respect to an orthonormal basis $\left(\boldsymbol{e}_{i}\right)$. The space of $n$ th-order tensors will be denoted by $\otimes^{n}\left(\mathbb{R}^{3}\right)$, and the subspace of totally symmetric tensors of order $n$ by $\mathbb{S}^{n}\left(\mathbb{R}^{3}\right)$. A traceless tensor $\mathbf{H} \in \mathbb{S}^{n}\left(\mathbb{R}^{3}\right)$ is called an harmonic tensor and the space of $n$ th-order harmonic tensors is denoted by $\mathbb{H}^{n}\left(\mathbb{R}^{3}\right)$.

The contraction over two or three indices between second/fourth-order tensors will be denoted by

$$
\begin{array}{ll}
\mathbf{a}: \mathbf{b}=a_{i j} b_{i j}, & (\mathbf{A}: \mathbf{a})_{i j}=A_{i j k l} a_{k l}, \\
(\mathbf{A}: \mathbf{B})_{i j k l}=A_{i j p q} B_{p q k l}, & (\mathbf{A}: \mathbf{B})_{i j}=A_{i p q r} B_{p q r j} .
\end{array}
$$

The total symmetrization of an $n$ th-order tensor $\mathbf{T}$ is the tensor $\mathbf{T}^{s}$, defined by

$$
\left(\mathbf{T}^{s}\right)_{i_{1} \ldots i_{n}}=\frac{1}{n !} \sum_{\sigma \in \mathfrak{S}_{n}} T_{i_{\sigma(1)} \ldots i_{\sigma(n)}} \in \mathbb{S}^{n}\left(\mathbb{R}^{3}\right),
$$

where $\mathfrak{S}_{n}$ is the permutation group over $n$ elements.

The symmetric tensor product, noted $\odot$, and the generalized cross product (introduced in [28]), noted $\times$, between two totally symmetric tensors $\mathbf{S}_{1} \in \mathbb{S}^{n_{1}}\left(\mathbb{R}^{3}\right)$ and $\mathbf{S}_{2} \in \mathbb{S}^{n_{2}}\left(\mathbb{R}^{3}\right)$, are defined respectively by

$$
\begin{aligned}
& \mathbf{S}_{1} \odot \mathbf{S}_{2}:=\left(\mathbf{S}_{1} \otimes \mathbf{S}_{2}\right)^{s} \in \mathbb{S}^{n_{1}+n_{2}}\left(\mathbb{R}^{3}\right), \\
& \mathbf{S}_{1} \times \mathbf{S}_{2}:=\left(\mathbf{S}_{2} \cdot \boldsymbol{\varepsilon} \cdot \mathbf{S}_{1}\right)^{s} \in \mathbb{S}^{n_{1}+n_{2}-1}\left(\mathbb{R}^{3}\right),
\end{aligned}
$$

where $\boldsymbol{\varepsilon}$ is the third-order Levi-Civita tensor (with components $\varepsilon_{i j k}=\operatorname{det}\left(\boldsymbol{e}_{i}, \boldsymbol{e}_{j}, \boldsymbol{e}_{k}\right)$ ). Explicit component formulas for the generalized cross product involving second and fourth-order tensors can be found in [1]. We have moreover [28]

$$
\mathbf{S} \times \mathbf{q}=0, \quad \forall \mathbf{S} \in \mathbb{S}^{n}\left(\mathbb{R}^{3}\right),
$$

where $\mathbf{q}=\left(\delta_{i j}\right)$ is the Euclidean metric. 


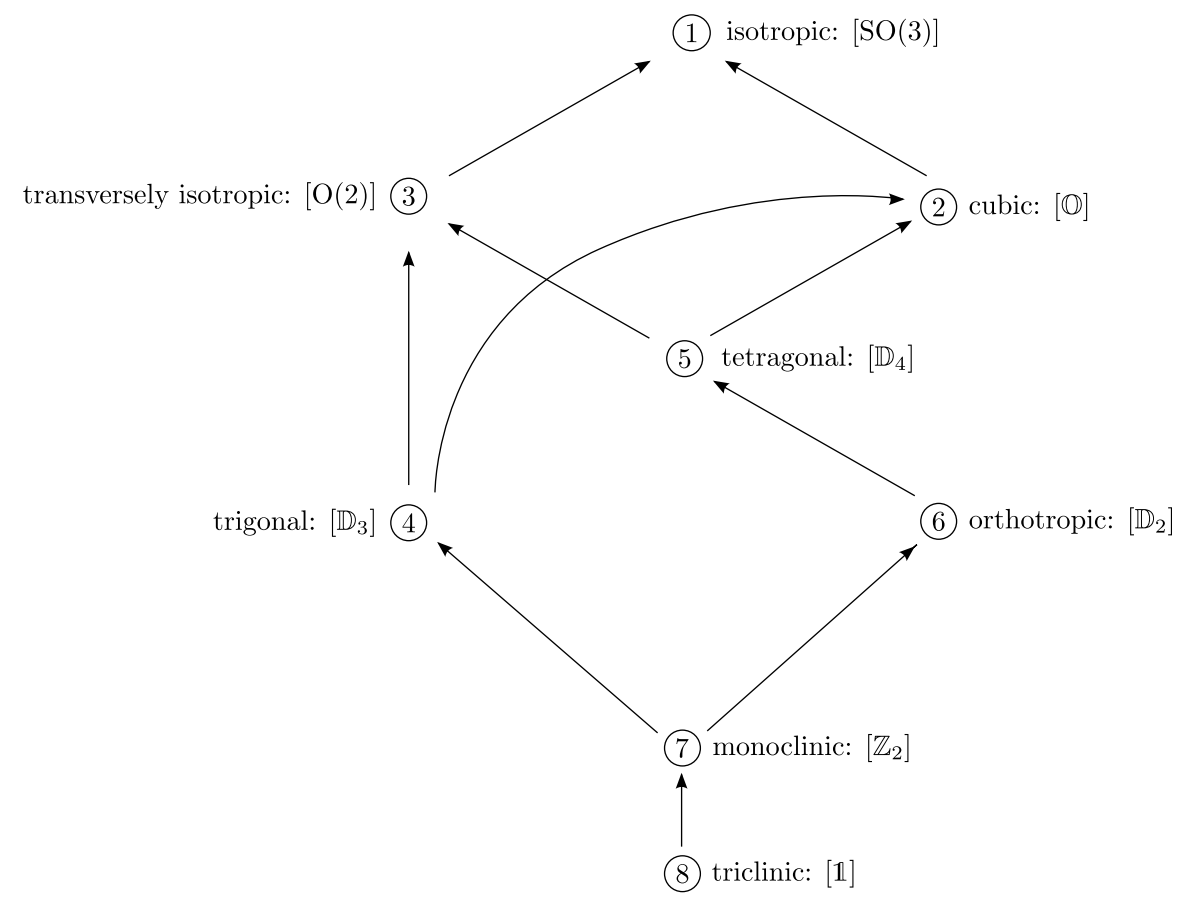

FiguRE 1. Symmetry classes of elasticity tensors and of fourth-order harmonic tensors $[20,17]$ (figure from [3]).

\section{Covariant Characterization of Elasticity SyMmetry Classes}

Let

$$
\mathbb{E l a}:=\left\{\mathbf{E} \in \otimes^{4}\left(\mathbb{R}^{3}\right) ; E_{i j k l}=E_{k l i j}=E_{j i k l}\right\}
$$

be the 21-dimensional vector space of three-dimensional elasticity tensors. It is endowed with the natural $\mathrm{SO}(3)$ representation given by

$$
(g \star \mathbf{E})_{i j k l}:=g_{i p} g_{j q} g_{k r} g_{l s} E_{i j k l}, \quad g \in \mathrm{SO}(3) .
$$

2.1. Elasticity symmetry classes and strata. Forte and Vianello [17] have shown that there are exactly eight different elasticity symmetry classes, depicted in Figure 1, and in which the mechanical names are provided aside the associate group designation $[H]$ : triclinic $[\mathbb{1}]$, monoclinic $\left[\mathbb{Z}_{2}\right]$, orthotropic $\left[\mathbb{D}_{2}\right]$, tetragonal $\left[\mathbb{D}_{4}\right]$, trigonal $\left[\mathbb{D}_{3}\right]$, transversely-isotropic $[\mathrm{O}(2)]$, cubic $[\mathbb{O}]$ and isotropic $[\mathrm{SO}(3)]$ (see Appendix A for the group notations).

Given a symmetry class $[H]$, the symmetry stratum $\Sigma_{[H]}$ is the set of all the elasticity tensors which have exactly the symmetry class $[H]$. Observe, for instance, that a transversely isotropic elasticity tensor $\mathbf{E}$ has also tetragonal symmetry. In such a case, we will say that $\mathbf{E}$ is at least tetragonal, but it does not belong to the tetragonal stratum $\Sigma_{\left[\mathbb{D}_{4}\right]}$. This "at least" order relation is depicted by the arrows of Figure 1.

2.2. Harmonic decomposition - Covariants. The first step, when studying the geometry of elasticity tensors, consists in splitting $\mathbb{E l a}$ into stable, irreducible vector spaces (under the action of $\mathrm{SO}(3)$ ). This is the so-called harmonic decomposition [4]. Introducing the second-order dilatation tensor

$$
\mathbf{d}:=\operatorname{tr}_{12} \mathbf{E}, \quad d_{i j}=E_{k k i j},
$$

and the second-order Voigt tensor

$$
\mathbf{v}:=\operatorname{tr}_{13} \mathbf{E}, \quad v_{i j}=E_{k i k j}
$$

one obtains an explicit harmonic decomposition of $\mathbf{E}$ (see $[14,14,5,17,1])$,

$$
\mathbf{E}=\left(\operatorname{tr} \mathbf{d}, \operatorname{tr} \mathbf{v}, \mathbf{d}^{\prime}, \mathbf{v}^{\prime}, \mathbf{H}\right) .
$$

In this decomposition, the harmonic components are the two scalar invariants

$\operatorname{tr} \mathbf{d}, \quad \operatorname{tr} \mathbf{v}$, 
the two deviatoric tensors

$$
\mathbf{d}^{\prime}=\mathbf{d}-\frac{1}{3}(\operatorname{tr} \mathbf{d}) \mathbf{q}, \quad \mathbf{v}^{\prime}=\mathbf{v}-\frac{1}{3}(\operatorname{tr} \mathbf{v}) \mathbf{q}
$$

and the harmonic (i.e., totally symmetric and traceless) fourth-order tensor

$$
\mathbf{H}=\mathbf{E}^{s}-\mathbf{q} \odot \mathbf{a}^{\prime}-\frac{7}{30}(\operatorname{tr} \mathbf{a}) \mathbf{q} \odot \mathbf{q}, \quad \mathbf{a}:=\frac{2}{7}(\mathbf{d}+2 \mathbf{v}),
$$

where $\mathbf{E}^{s}$ is the totally symmetric part of $\mathbf{E}$, and $\odot$ is the symmetrized tensor product defined in (1.1). The harmonic decomposition (2.2) is equivariant, meaning that it satisfies:

$$
g \star \mathbf{E}=\left(g \star \operatorname{tr} \mathbf{d}, g \star \operatorname{tr} \mathbf{v}, g \star \mathbf{d}^{\prime}, g \star \mathbf{v}^{\prime}, g \star \mathbf{H}\right)=\left(\operatorname{tr} \mathbf{d}, \operatorname{tr} \mathbf{v}, g \star \mathbf{d}^{\prime}, g \star \mathbf{v}^{\prime}, g \star \mathbf{H}\right),
$$

for any rotation $g \in \mathrm{SO}(3)$. Note here that $g \star \lambda=\lambda$ for scalar invariants $\lambda$. The action of a rotation on a second-order tensor $\mathbf{a}$ is $g \star \mathbf{a}=g \mathbf{a} g^{t}$, while the action of a rotation on a fourth-order tensor is given by (2.1). The harmonic components

$$
\operatorname{tr} \mathbf{d}=\operatorname{tr}(\mathbf{d}(\mathbf{E})), \quad \operatorname{tr} \mathbf{v}=\operatorname{tr}(\mathbf{v}(\mathbf{E})), \quad \mathbf{d}^{\prime}=\mathbf{d}^{\prime}(\mathbf{E}), \quad \mathbf{v}^{\prime}=\mathbf{v}^{\prime}(\mathbf{E}), \quad \mathbf{H}=\mathbf{H}(\mathbf{E}),
$$

are covariants $\mathbf{C}(\mathbf{E})$ of $\mathbf{E}[21,29]$ (of respective order 0, 0, 2, 2 and 4, tr $\mathbf{d}$ and tr $\mathbf{v}$ being scalar invariants of $\mathbf{E}$, and $\mathbf{d}^{\prime}(\mathbf{E}), \mathbf{v}^{\prime}(\mathbf{E})$ and $\mathbf{H}=\mathbf{H}(\mathbf{E})$ being linear covariants of $\left.\mathbf{E}\right)$. They satisfy the rule

$$
\mathbf{C}(g \star \mathbf{E})=g \star \mathbf{C}(\mathbf{E}), \quad \forall g \in \mathrm{SO}(3) .
$$

However, there also exists polynomial covariants of higher degree. For instance, the quadratic covariant

$$
\mathbf{d}_{2}(\mathbf{H}):=\mathbf{H}: \mathbf{H}, \quad\left(\text { i.e. },\left(\mathbf{d}_{2}\right)_{i j}=H_{i p q r} H_{p q r j}\right),
$$

introduced by Boehler, Kirillov and Onat in 1994 [7], and which plays a fundamental role in the classification (by symmetry classes) of the fourth-order harmonic tensor and of the elasticity tensor. Indeed, necessary and sufficient conditions for an elasticity tensor to be of a given symmetry class have been formulated in [29], involving $\mathbf{d}, \mathbf{v}, \mathbf{d}_{2}$ and other higher degree polynomial covariants.

2.3. Covariant characterization of elasticity symmetry classes. The following theorem was proved in [29, Theorem 10.2]. It provides a characterization of the isotropic, cubic, transversely isotropic, tetragonal and trigonal symmetry classes of elasticity (that is for elasticity tensors which are at least trigonal or tetragonal). We denote by $\mathbf{a}^{\prime}=\mathbf{a}-\frac{1}{3}(\operatorname{tr} \mathbf{a}) \mathbf{q}$, the deviatoric part of a symmetric second-order tensor a and recall that $\mathbf{H} \times \mathbf{q}=0$, so that $\mathbf{H} \times \mathbf{a}=\mathbf{H} \times \mathbf{a}^{\prime}$.

Theorem 2.1. Let $\mathbf{E}=\left(\operatorname{tr} \mathbf{d}, \operatorname{tr} \mathbf{v}, \mathbf{d}^{\prime}, \mathbf{v}^{\prime}, \mathbf{H}\right) \in \mathbb{E}$ la be an elasticity tensor. Then

(1) $\mathbf{E}$ is isotropic if and only if $\mathbf{d}^{\prime}=\mathbf{v}^{\prime}=\mathbf{d}_{2}=0$.

(2) $\mathbf{E}$ is cubic if and only if $\mathbf{d}^{\prime}=\mathbf{v}^{\prime}=\mathbf{d}_{2}^{\prime}=0$ and $\mathbf{d}_{2} \neq 0$.

(3) $\mathbf{E}$ is transversely isotropic if and only if $\left(\mathbf{d}_{2}, \mathbf{d}, \mathbf{v}\right)$ is transversely isotropic and

$$
\mathbf{H} \times \mathbf{d}_{2}=\mathbf{H} \times \mathbf{d}=\mathbf{H} \times \mathbf{v}=0 .
$$

(4) $\mathbf{E}$ is tetragonal if and only if $\left(\mathbf{d}_{2}, \mathbf{d}, \mathbf{v}\right)$ is transversely isotropic,

$$
\operatorname{tr}\left(\mathbf{H} \times \mathbf{d}_{2}\right)=\operatorname{tr}(\mathbf{H} \times \mathbf{d})=\operatorname{tr}(\mathbf{H} \times \mathbf{v})=0,
$$

and

$$
\mathbf{H} \times \mathbf{d}_{2} \neq 0, \quad \text { or } \quad \mathbf{H} \times \mathbf{d} \neq 0, \quad \text { or } \quad \mathbf{H} \times \mathbf{v} \neq 0 .
$$

(5) $\mathbf{E}$ is trigonal if and only if $\left(\mathbf{d}_{2}, \mathbf{d}, \mathbf{v}\right)$ is transversely isotropic,

$$
\mathbf{d}_{2} \times\left(\mathbf{H}: \mathbf{d}_{2}\right)=\mathbf{d} \times(\mathbf{H}: \mathbf{d})=\mathbf{v} \times(\mathbf{H}: \mathbf{v})=0,
$$

and

$$
\operatorname{tr}\left(\mathbf{H} \times \mathbf{d}_{2}\right) \neq 0, \quad \text { or } \quad \operatorname{tr}(\mathbf{H} \times \mathbf{d}) \neq 0, \quad \text { or } \quad \operatorname{tr}(\mathbf{H} \times \mathbf{v}) \neq 0 .
$$

As a corollary of this theorem, we have the following result.

Corollary 2.2. Let $\mathbf{E}$ be an elasticity tensor which is either transversely isotropic, tetragonal or trigonal. Then, $\left(\mathbf{d}, \mathbf{v}, \mathbf{d}_{2}\right)$ is transversely isotropic (or equivalently $\left(\mathbf{d}^{\prime}, \mathbf{v}^{\prime}, \mathbf{d}_{2}^{\prime}\right)$ is transversely isotropic). In particular, there exists a unit vector $\boldsymbol{n}$, defining the axis $\langle\boldsymbol{n}\rangle$ of transverse isotropy, and such that

$$
\mathbf{d}^{\prime}=\alpha(\boldsymbol{n} \otimes \boldsymbol{n})^{\prime}, \quad \mathbf{v}^{\prime}=\beta(\boldsymbol{n} \otimes \boldsymbol{n})^{\prime}, \quad \mathbf{d}_{2}^{\prime}=\gamma(\boldsymbol{n} \otimes \boldsymbol{n})^{\prime},
$$

where $(\alpha, \beta, \gamma) \neq(0,0,0)$. 


\section{FunCtional BASES AND SEPARATING SETS}

In this section, we recall basic notions in Invariant Theory, in particular: functional basis, separating set and integrity basis, and the associated notion of minimality. The concepts of functional basis and separating set are meaningful in a very general setting, namely for the action of a group $G$ on a set $\mathrm{X}$ [42], and are moreover equivalent, as noted by Wineman and Pipkin [43]. Defining a finite integrity basis requires some additional structure, for instance that $G$ is a compact Lie group [11] (with the remark that in solid mechanics, many relevant groups are compact), $\mathrm{X}=\mathrm{V}$ is a vector space, and the action of $G$ on $\mathrm{V}$ is linear.

3.1. Action of a group on a set. An action $\star$ of a group $G$ on a set $\mathrm{X}$ is a mapping

$$
G \times \mathrm{X} \rightarrow \mathrm{X}, \quad(g, x) \mapsto g \star x,
$$

such that

$$
\left(g_{1} g_{2}\right) \star x=g_{1} \star\left(g_{2} \star x\right), \quad e \star x=x,
$$

where $g_{1}, g_{2} \in G$ and $e$ is the unit element of $G$. When $\mathrm{X}=\mathrm{V}$ is a vector space and the action is linear in $x$, such an action is called a linear representation of $G$ on $\mathrm{X}$. The symmetry group of $x$ (also known as the isotropy group of $x$ ) is defined as $G_{x}:=\{g \in G, g \star x=x\}$ and the symmetry class of $x$, noted $\left[G_{x}\right]$, is defined as the conjugacy class of $G_{x}$ in $G$, i.e.

$$
\left[G_{x}\right]:=\left\{g G_{x} g^{-1}, g \in G\right\} .
$$

A symmetry stratum $\Sigma_{[H]}$ is the set of all elements $x$ with symmetry group $G_{x}$ conjugate to $H$ :

$$
\Sigma_{[H]}:=\left\{x \in X, \quad G_{x} \in[H]\right\} .
$$

The orbit of the point $x \in \mathrm{X}$ is defined as the set

$$
\operatorname{Orb}(x):=\{g \star x, \quad g \in G\} .
$$

Observe that all points in $\operatorname{Orb}(x)$ belong to the same symmetry stratum, since $G_{g \star x}=g G_{x} g^{-1}$. Finally, the orbit space $\mathrm{X} / G$ is the set of orbits and the canonical projection is the mapping

$$
\pi: \mathrm{X} \longrightarrow \mathrm{X} / G, \quad x \mapsto \operatorname{Orb}(x) .
$$

3.2. Functional bases and separating sets. The action of $G$ on $\mathrm{X}$ induces a linear action of $G$ on the vector space $\mathcal{F}(\mathrm{X})$ of real-valued functions on $\mathrm{X}$, which is written

$$
(g \star f)(x):=f\left(g^{-1} \star x\right),
$$

where $f \in \mathcal{F}(\mathrm{X})$ and $g \in G$. The algebra $\mathcal{F}(\mathrm{X})^{G}$ of $G$-invariant functions on $\mathrm{X}$ is defined by

$$
\mathcal{F}(\mathrm{X})^{G}:=\{f \in \mathcal{F}(\mathrm{X}), \quad g \star f=f, \quad \forall g \in G\},
$$

and this definition leads to the notion of functional basis for $G$-invariant functions on X. This notion, introduced in Weyl's classical book [42], has become a key notion in the mechanical science literature related to Invariant Theory $[43,35,6,44]$.

Definition 3.1 (Functional basis). A finite set $\mathscr{F}:=\left\{\varphi_{1}, \ldots, \varphi_{s}\right\}$ of $G$-invariant functions is a functional basis of $\mathcal{F}(\mathrm{X})^{G}$ if for any $G$-invariant function $f \in \mathcal{F}(\mathrm{X})^{G}$ there exists a function $F: \mathbb{R}^{s} \rightarrow \mathbb{R}$ such that

$$
f(x)=F\left(\varphi_{1}(x), \ldots, \varphi_{s}(x)\right), \quad \forall x \in \mathrm{X} .
$$

A functional basis $\mathscr{F}$ is said to be minimal if no proper subset $\mathscr{F}^{\prime}$ of $\mathscr{F}$ is a functional basis.

As pointed out by Weyl [42, Page 30], the word function has to be understood in its widest scope. Such a function $F$ may not even be continuous [35, Section 5].

Definition 3.2 (Separating set). A finite set $\mathscr{S}:=\left\{\kappa_{1}, \ldots, \kappa_{r}\right\}$ of $G$-invariant functions is a separating set of $\mathrm{X} / G$ if for any $x, \bar{x}$ in $\mathrm{X}$

$$
\operatorname{Orb}(x)=\operatorname{Orb}(\bar{x}) \Longleftrightarrow \kappa_{i}(x)=\kappa_{i}(\bar{x}), \quad i=1, \ldots, r .
$$

A separating set $\mathscr{S}$ is said to be minimal if no proper subset $\mathscr{S}^{\prime}$ of $\mathscr{S}$ is a separating set.

Given a separating set $\left\{\kappa_{1}, \ldots, \kappa_{r}\right\}$ of invariant functions, the mapping

$$
K: \mathrm{X} \longrightarrow \mathbb{R}^{r}, \quad x \mapsto\left(\kappa_{1}(x), \ldots, \kappa_{r}(x)\right) .
$$

induces an injective mapping from the orbit space $\mathrm{X} / G$ into $\mathbb{R}^{r}$ and one has the following result [43] (see also $[31,32])$. 
Theorem 3.3 (Wineman and Pipkin). Consider a group $G$ acting on a set $\mathrm{X}$. Then, each separating set $\left\{\kappa_{1}, \ldots, \kappa_{r}\right\}$ of $\mathrm{X} / G$ is a functional basis of $\mathcal{F}(\mathrm{X})^{G}$ : for each $G$-invariant function $f$, there exists a function

$$
F: \operatorname{Im}(K) \longrightarrow \mathbb{R}, \quad \operatorname{Im}(K):=\{K(x) ; x \in \mathrm{X}\}
$$

such that

$$
f(x)=F\left(\kappa_{1}(x), \cdots, \kappa_{r}(x)\right), \quad \forall x \in \mathbf{X} .
$$

Conversely, each functional basis $\mathscr{F}=\left\{\varphi_{1}, \ldots, \varphi_{s}\right\}$ of $\mathcal{F}(\mathrm{X})^{G}$ is also a separating set of $\mathrm{X} / G$.

Note that the cardinality of a minimal separating set/functional basis is not well-defined. It may vary from one minimal set to another. Besides, a lower bound on the cardinality of such a set depends drastically on the class of functions (continuous, differentiable, ...) for which it is defined. For instance, Wang [41] (see also [6, p.39]) has noticed that, by omitting continuity, it is always possible to construct a separating set of only one element. On the other side, if X/G is (at least) a topological manifold and the class of invariant functions considered are at least continuous, then the cardinality of a functional basis is at least the dimension of the quotient space $\mathrm{X} / G$, as detailed in the following remark.

Remark 3.4. When the orbit space $\mathrm{X} / G$ is a topological manifold of dimension $d$, the cardinality of any separating set $\left\{\kappa_{1}, \ldots, \kappa_{r}\right\}$ of continuous functions is bigger than the dimension of $\mathrm{X} / G(r \geq d)$. This is a consequence of the invariance of domain theorem $[12,19]$, which states that if there is a continuous injective mapping $f$ from an open subset $U$ of $\mathbb{R}^{d}$ into $\mathbb{R}^{r}$, then, necessarily $r \geq d$.

3.3. Linear representation of a compact Lie group. From now on, we focus on a linear action of a compact Lie group $G$ on a vector space V (usually called a linear representation of $G$ on $\mathrm{V}$ ). In that case, there exists only a finite number of symmetry classes $\left[H_{1}\right], \ldots,\left[H_{l}\right]$ and $\mathrm{V}$ splits into a disjoint union of strata $[2,9]$

$$
\mathrm{V}=\Sigma_{\left[H_{1}\right]} \cup \ldots \cup \Sigma_{\left[H_{l}\right]},
$$

where each stratum $\Sigma_{[H]}$ is a $G$-stable smooth submanifold of $\mathrm{V}[10,2,30,3]$.

We shall denote by $\mathbb{R}[\mathrm{V}]$, the algebra of polynomial functions on $\mathrm{V}$, and by

$$
\mathbb{R}[\mathrm{V}]^{G}:=\{\mathrm{p} \in \mathbb{R}[\mathrm{V}] ; \mathrm{p}(g \star \boldsymbol{v})=\mathrm{p}(\boldsymbol{v}), \quad \forall g \in G, \forall \boldsymbol{v} \in \mathrm{V}\},
$$

the subalgebra of $\mathbb{R}[\mathrm{V}]$ consisting of polynomial invariants. As a consequence of Hilbert's finiteness theorem $[18,36]$, the algebra $\mathbb{R}[\mathrm{V}]^{G}$ is finitely generated and any finite set $\left\{I_{1}, \ldots, I_{N}\right\}$ of generators is called an integrity basis. We recall that the generating property means that each $G$-invariant polynomial $J \in \mathbb{R}[\mathrm{V}]^{G}$ is a polynomial function in $I_{1}, \ldots, I_{N}$ :

$$
J(\boldsymbol{v})=\mathrm{p}\left(I_{1}(\boldsymbol{v}), \ldots, I_{N}(\boldsymbol{v})\right), \quad \boldsymbol{v} \in \mathrm{V},
$$

where $\mathrm{p}$ is a polynomial in $N$ variables. An integrity basis is minimal if no proper subset of it is an integrity basis.

As we are dealing with linear representations of a compact Lie group on a real vector space, any integrity basis is also a separating set of the orbit space $\mathrm{V} / G$ (see $[2$, Appendix $\mathrm{C}]$ ), and is thus a functional basis of $\mathcal{F}(\mathrm{V})^{G}$.

We will end this section by formulating a theorem which will be helpful to achieve our goal which is to produce minimal functional bases for the stable subsets $\Sigma_{[H]}$ of $\mathrm{V}$, rather than for $\mathrm{V}$ itself.

Theorem 3.5. Let $\mathcal{B}:=\left\{I_{1}, \ldots, I_{N}\right\}$ be an integrity basis of $\mathbb{R}[\mathrm{V}]^{G}$, and $\Sigma_{[H]}$, a symmetry stratum with $d=\operatorname{dim}\left(\Sigma_{[H]} / G\right)$. Suppose that there exist $G$-invariant continuous functions $\kappa_{1}, \ldots, \kappa_{d}$ in $\mathcal{F}\left(\Sigma_{[H]}\right)^{G}$ and functions $F_{1}, \ldots, F_{N}$ such that

$$
I_{k}(\boldsymbol{v})=F_{k}\left(\kappa_{1}(\boldsymbol{v}), \ldots, \kappa_{d}(\boldsymbol{v})\right), \quad \forall \boldsymbol{v} \in \Sigma_{[H]}, \quad \forall k=1, \ldots, N .
$$

Then $\left\{\kappa_{1}, \ldots, \kappa_{d}\right\}$ is a minimal separating set of $\Sigma_{[H]} / G$ and a minimal functional basis of $\mathcal{F}\left(\Sigma_{[H]}\right)^{G}$.

Proof. As already noticed, for a real representation of a compact Lie group, an integrity basis $\mathcal{B}$ is also a separating set of $\mathrm{V} / G\left[2\right.$, Appendix C]. By hypothesis, for any $\boldsymbol{v}, \overline{\boldsymbol{v}} \in \Sigma_{[H]}$

$$
\forall i, \quad \kappa_{i}(\boldsymbol{v})=\kappa_{i}(\overline{\boldsymbol{v}}) \Longrightarrow \forall k, \quad I_{k}(\boldsymbol{v})=I_{k}(\overline{\boldsymbol{v}}) .
$$

Hence, $\operatorname{Orb}(\boldsymbol{v})=\operatorname{Orb}(\overline{\boldsymbol{v}})$, and we deduce that the set $\left\{\kappa_{1}, \ldots, \kappa_{d}\right\}$ is a separating set of $\Sigma_{[H]} / G$, as well as a functional basis of $\mathcal{F}\left(\Sigma_{[H]}\right)^{G}$ by theorem 3.3. Finally, the minimality is a direct consequence of remark 3.4 . 


\section{FunCtional BASES ON SYMMETRY STRATA OF SECOND-ORDER TENSORS}

Let us first illustrate the notions introduced in section 3 for the standard action of the rotation group $G=\mathrm{SO}(3)$ on the vector space $\mathrm{V}=\mathbb{S}^{2}\left(\mathbb{R}^{3}\right)$ of symmetric second-order tensors on $\mathbb{R}^{3}$. The action is written $g \star \mathbf{a}:=g \mathbf{a} g^{t}$ and there are three different symmetry classes (orthotropic $\left[\mathbb{D}_{2}\right]$, transversely isotropic $[\mathrm{O}(2)]$ and isotropic $[\mathrm{SO}(3)]$, see Appendix A for group definitions). The three corresponding symmetry strata $\Sigma_{\left[\mathbb{D}_{2}\right]}, \Sigma_{[\mathrm{O}(2)]}$ and $\Sigma_{[\mathrm{SO}(3)]}$, are characterized by polynomial equations. These conditions can be formulated, as algebraic equations involving either polynomial invariants, or polynomial covariants [21].

Each second-order tensor $\mathbf{a} \in \mathbb{S}^{2}\left(\mathbb{R}^{3}\right)$ splits as $\mathbf{a}=\mathbf{a}^{\prime}+\frac{1}{3}(\operatorname{tr} \mathbf{a}) \mathbf{q}$, where the deviatoric part $\mathbf{a}^{\prime}$ is a polynomial (linear) covariant of $\mathbf{a}$, meaning that $\mathbf{a}^{\prime}$ is expressed polynomially (linearly) in the $a_{i j}$, and that for any $g \in \mathrm{SO}(3)$,

$$
(g \star \mathbf{a})^{\prime}=g \star \mathbf{a}^{\prime} .
$$

A less common but very important polynomial covariant of a was obtained in [29] using the generalized cross product (1.1),

$$
\mathbf{S}(\mathbf{a}):=\mathbf{a} \times \mathbf{a}^{2} \in \mathbb{S}^{3}\left(\mathbb{R}^{3}\right), \quad \text { with } \quad g \star\left(\mathbf{a} \times \mathbf{a}^{2}\right)=(g \star \mathbf{a}) \times(g \star \mathbf{a})^{2},
$$

for any rotation $g$.

The algebraic equations characterizing each symmetry stratum of $\mathbb{S}^{2}\left(\mathbb{R}^{3}\right)$ are stated in table 1 , where we consider the three following polynomial invariants

$$
I_{1}:=\operatorname{tr} \mathbf{a}, \quad J_{2}:=\operatorname{tr}\left(\mathbf{a}^{\prime 2}\right), \quad J_{3}:=\operatorname{tr}\left(\mathbf{a}^{\prime 3}\right),
$$

which constitute a minimal integrity basis of $\mathbb{R}\left[\mathbb{S}^{2}\left(\mathbb{R}^{3}\right)\right]^{\mathrm{SO}(3)}$.

Remark 4.1. The characterization conditions using covariants are of degree (in a) half the degree of those using invariants. Indeed

$$
J_{2}=\left\|\mathbf{a}^{\prime}\right\|^{2}, \quad J_{2}^{3}-6 J_{3}^{2}=12\left\|\mathbf{a} \times \mathbf{a}^{2}\right\|^{2} .
$$

\begin{tabular}{|c||c|c|}
\hline Stratum & Conditions in terms of invariants & Conditions in terms of covariants \\
\hline \hline$\Sigma_{\left[\mathbb{D}_{2}\right]}$ & $J_{2}^{3}-6 J_{3}^{2} \neq 0$ & $\mathbf{a} \times \mathbf{a}^{2} \neq \mathbf{0}$ \\
$\Sigma_{[\mathrm{O}(2)]}$ & $J_{2}^{3}-6 J_{3}^{2}=0$ and $J_{2} \neq 0$ & $\mathbf{a} \times \mathbf{a}^{2}=\mathbf{0}$ and $\mathbf{a}^{\prime} \neq \mathbf{0}$ \\
$\Sigma_{[\mathrm{SO}(3)]}$ & $J_{2}=0$ & $\mathbf{a}^{\prime}=\mathbf{0}$ \\
\hline
\end{tabular}

TABLE 1. Algebraic equations defining the symmetry strata of $\mathbb{S}^{2}\left(\mathbb{R}^{3}\right)[29]$.

In contrast to the entire orbit space $\mathrm{V} / G$, each orbit space $\Sigma_{[H]} / G$ is a smooth manifold $[2,10,30]$ and when $\mathrm{V}=\mathbb{S}^{2}\left(\mathbb{R}^{3}\right)$ we have:

$$
\operatorname{dim}\left(\Sigma_{\left[\mathbb{D}_{2}\right]} / \mathrm{SO}(3)\right)=3, \quad \operatorname{dim}\left(\Sigma_{[\mathrm{O}(2)]} / \mathrm{SO}(3)\right)=2, \quad \operatorname{dim}\left(\Sigma_{[\mathrm{SO}(3)]} / \mathrm{SO}(3)\right)=1 .
$$

Next, we will show how theorem 3.5 helps us to obtain minimal functional bases for the orthotropic $\left(\Sigma_{\left[\mathbb{D}_{2}\right]}\right)$ and the transversely isotropic $\left(\Sigma_{[\mathrm{O}(2)]}\right)$ strata.

4.1. Orthotropic stratum. The orbit space $\Sigma_{\left[\mathbb{D}_{2}\right]} / \mathrm{SO}(3)$ is three dimensional. An integrity basis is also a separating set [2, Appendix C], and by the Wineman-Pipkin theorem 3.3, it is also a functional basis. Thus, the set (4.1), satisfying the hypotheses of theorem 3.5, is an example of application of this theorem, which is formulated below.

Lemma 4.2. A minimal functional basis for $\Sigma_{\left[\mathbb{D}_{2}\right]}$, i.e., for orthotropic second-order tensors, consists of the three polynomial invariants

$$
\kappa_{1}:=I_{1}=\operatorname{tr} \mathbf{a}, \quad \kappa_{2}:=J_{2}=\operatorname{tr}\left(\mathbf{a}^{\prime 2}\right), \quad \kappa_{3}:=J_{3}=\operatorname{tr}\left(\mathbf{a}^{\prime 3}\right) .
$$

4.2. Transversely isotropic stratum. In this case, we first note that a second-order tensor $\mathbf{a}$ is in the symmetry stratum $\Sigma_{[\mathrm{O}(2)]}$ if and only if there exists a rotation $g \in \mathrm{SO}(3)$ such that $\mathbf{a}=g \star \mathbf{a}_{0}$, where $\mathbf{a}_{0}$ is

$$
\mathbf{a}_{0}=\left(\begin{array}{ccc}
\delta_{1}-\delta_{2} & 0 & 0 \\
0 & \delta_{1}-\delta_{2} & 0 \\
0 & 0 & \delta_{1}+2 \delta_{2}
\end{array}\right), \quad \delta_{2} \neq 0,
$$

in the orthonormal basis $\left(\boldsymbol{e}_{i}\right)$. The condition $\delta_{2} \neq 0$ means that $\mathbf{a}_{0}$ is genuinely transversely isotropic (and not isotropic). Moreover its symmetry group is the subgroup $\mathrm{O}(2)$ of $\mathrm{SO}(3)$ defined in Appendix A. 
Lemma 4.3. A minimal functional basis for $\Sigma_{[\mathrm{O}(2)]}$, i.e., for transversely isotropic symmetric secondorder tensors, consists of the two rational invariants

$$
\kappa_{1}:=I_{1}, \quad \kappa_{2}:=\frac{J_{3}}{J_{2}} .
$$

Proof. Evaluating the invariants $J_{2}$ and $J_{3}$ on (4.2), we get

$$
J_{2}(\mathbf{a})=6 \delta_{2}^{2}, \quad J_{3}(\mathbf{a})=6 \delta_{2}^{3}, \quad \delta_{2} \neq 0,
$$

and hence $\kappa_{2}(\mathbf{a})=\delta_{2}$. We have therefore

$$
I_{1}(\mathbf{a})=\kappa_{1}(\mathbf{a}), \quad J_{2}(\mathbf{a})=6 \kappa_{2}^{2}(\mathbf{a}), \quad J_{3}(\mathbf{a})=6 \kappa_{2}^{3}(\mathbf{a}),
$$

and the result follows by theorem 3.5 applied to $\mathrm{V}=\mathbb{S}^{2}\left(\mathbb{R}^{3}\right)$ and the symmetry stratum $\Sigma_{[\mathrm{O}(2)]}$, with $\operatorname{dim}\left(\Sigma_{[\mathrm{O}(2)]} / \mathrm{SO}(3)\right)=2$.

The rational invariants $\kappa_{1}, \kappa_{2}$ in lemma 4.3 can be considered as global parameters of $\mathrm{X}=\Sigma_{[\mathrm{O}(2)]} / \mathrm{SO}(3)$.

Proposition 4.4. Any transversely isotropic second-order symmetric tensor $\mathbf{a} \in \Sigma_{[\mathrm{O}(2)]}$ can be written

$$
\mathbf{a}=\frac{1}{3} \kappa_{1} \mathbf{q}+3 \kappa_{2} \mathbf{t}, \quad \kappa_{1}:=I_{1}, \quad \kappa_{2}:=\frac{J_{3}}{J_{2}}=\frac{\operatorname{sgn}\left(J_{3}\right)}{\sqrt{6}}\left\|\mathbf{a}^{\prime}\right\|,
$$

with $\mathbf{t}:=(\boldsymbol{n} \otimes \boldsymbol{n})^{\prime},\|\boldsymbol{n}\|=1$, where the vector $\boldsymbol{n}$ defines the axis of transverse isotropy and $\operatorname{sgn}(x)=x /|x|$ is the sign function.

\section{FunCtional BASES ON SYMMETRY STRATA OF HARMONiC FOURTH-ORDER TENSORS}

Let us now consider the vector space of fourth-order harmonic tensors in $\mathbb{R}^{3}$

$$
\mathbb{H}^{4}\left(\mathbb{R}^{3}\right):=\left\{\mathbf{H} \in \mathbb{S}^{4}\left(\mathbb{R}^{3}\right), \quad \operatorname{tr} \mathbf{H}=0\right\},
$$

i.e., of traceless totally symmetric fourth-order tensors. It is of dimension nine and appears as an irreducible subspace in the harmonic decomposition of $\mathbb{E}$ la. Its structure is more tricky than the one of $\mathbb{H}^{2}\left(\mathbb{R}^{3}\right)$ and has been investigated in [17] and [3, 29, 28]. The eight symmetry classes $[H]$ for $\mathbb{H}^{4}\left(\mathbb{R}^{3}\right)$ are the same as for $\mathbb{E}$ la (see Figure 1, Appendix B). Each orbit space $\Sigma_{[H]} / \mathrm{SO}(3)$ is a smooth manifold, and (see [3], for instance)

$$
\begin{array}{lll}
\operatorname{dim}\left(\Sigma_{[\mathbb{1}]} / \mathrm{SO}(3)\right)=6, & \operatorname{dim}\left(\Sigma_{\left[\mathbb{Z}_{2}\right]} / \mathrm{SO}(3)\right)=5, & \operatorname{dim}\left(\Sigma_{\left[\mathbb{D}_{2}\right]} / \mathrm{SO}(3)\right)=3, \\
\operatorname{dim}\left(\Sigma_{\left[\mathbb{D}_{4}\right]} / \mathrm{SO}(3)\right)=2, & \operatorname{dim}\left(\Sigma_{\left[\mathbb{D}_{3}\right]} / \mathrm{SO}(3)\right)=2, & \operatorname{dim}\left(\Sigma_{[\mathrm{O}(2)]} / \mathrm{SO}(3)\right)=1, \\
\operatorname{dim}\left(\Sigma_{[\mathbb{O}]} / \mathrm{SO}(3)\right)=1, & \operatorname{dim}\left(\Sigma_{[\mathrm{SO}(3)]} / \mathrm{SO}(3)\right)=0 . &
\end{array}
$$

A minimal integrity basis of nine polynomial invariants for the invariant algebra of $\mathbb{H}^{4}\left(\mathbb{R}^{3}\right)$, has been derived by Boehler, Kirillov and Onat [7], using previous works on binary forms by Shioda [34]. An alternative minimal integrity basis has been proposed in [15, Theorem 2.7]. It involves only the two second-order covariants $\mathbf{d}_{2}$ and $\mathbf{d}_{3}[7]$

$$
\mathbf{d}_{2}:=\operatorname{tr}_{13} \mathbf{H}^{2}, \quad \mathbf{d}_{3}:=\operatorname{tr}_{13} \mathbf{H}^{3},
$$

which, in components write

$$
\left(\mathbf{d}_{2}\right)_{i j}=H_{i p q r} H_{p q r j}, \quad \text { and } \quad\left(\mathbf{d}_{3}\right)_{i j}=H_{i k p q} H_{p q r s} H_{r s k j} .
$$

Here, we shall work with a slightly modified integrity basis,

$$
\begin{array}{lll}
I_{2}:=\operatorname{tr} \mathbf{d}_{2}, & I_{3}:=\operatorname{tr} \mathbf{d}_{3}, & I_{4}:=\operatorname{tr} \mathbf{d}_{2}^{\prime 2}, \\
I_{5}:=\operatorname{tr}\left(\mathbf{d}_{2}^{\prime} \mathbf{d}_{3}^{\prime}\right), & I_{6}:=\operatorname{tr} \mathbf{d}_{2}^{\prime 3}, & I_{7}:=\operatorname{tr}\left(\mathbf{d}_{2}^{\prime 2} \mathbf{d}_{3}^{\prime}\right), \\
I_{8}:=\operatorname{tr}\left(\mathbf{d}_{2}^{\prime} \mathbf{d}_{3}^{\prime 2}\right), & I_{9}:=\operatorname{tr} \mathbf{d}_{3}^{\prime 3}, & I_{10}:=\operatorname{tr}\left(\mathbf{d}_{2}^{\prime 2} \mathbf{d}_{3}^{\prime 2}\right) .
\end{array}
$$

In the following, we consider Kelvin's representation of a fourth-order harmonic tensor $\mathbf{H}=\left(H_{i j k l}\right)$, i.e., in an orthonormal basis, the symmetric matrix $[37,33]$

$$
[\mathbf{H}]:=\left(\begin{array}{cccccc}
H_{1111} & H_{1122} & H_{1133} & \sqrt{2} H_{1123} & \sqrt{2} H_{1113} & \sqrt{2} H_{1112} \\
H_{1122} & H_{2222} & H_{2233} & \sqrt{2} H_{2223} & \sqrt{2} H_{1223} & \sqrt{2} H_{1222} \\
H_{1133} & H_{2233} & H_{3333} & \sqrt{2} H_{2333} & \sqrt{2} H_{1333} & \sqrt{2} H_{1233} \\
\sqrt{2} H_{1123} & \sqrt{2} H_{2223} & \sqrt{2} H_{2333} & 2 H_{2233} & 2 H_{1233} & 2 H_{1223} \\
\sqrt{2} H_{1113} & \sqrt{2} H_{1223} & \sqrt{2} H_{1333} & 2 H_{1233} & 2 H_{1133} & 2 H_{1123} \\
\sqrt{2} H_{1112} & \sqrt{2} E_{1222} & \sqrt{2} H_{1233} & 2 H_{1223} & 2 H_{1123} & 2 H_{1122}
\end{array}\right)
$$


with $9\left(=\operatorname{dim} \mathbb{H}^{4}\left(\mathbb{R}^{3}\right)\right)$ independent components since

$$
\begin{array}{lll}
H_{1111}=-H_{1122}-H_{1133}, & H_{2222}=-H_{1122}-H_{2233}, & H_{3333}=-H_{1133}-H_{2233}, \\
H_{2333}=-H_{1123}-H_{2223}, & H_{1113}=-H_{1223}-H_{1333}, & H_{1222}=-H_{1112}-H_{1233} .
\end{array}
$$

5.1. Cubic stratum. A fourth-order tensor $\mathbf{H} \in \mathbb{H}^{4}\left(\mathbb{R}^{3}\right)$ is at least cubic if and only if there exists a rotation $g \in \mathrm{SO}(3)$ such that $\mathbf{H}=g \star \mathbf{H}_{\mathbb{O}}$, where $\mathbf{H}_{\mathbb{O}}$ has the following Kelvin representation [3],

$$
\left[\mathbf{H}_{\mathbb{O}}\right]=\left(\begin{array}{cccccc}
8 \delta & -4 \delta & -4 \delta & 0 & 0 & 0 \\
-4 \delta & 8 \delta & -4 \delta & 0 & 0 & 0 \\
-4 \delta & -4 \delta & 8 \delta & 0 & 0 & 0 \\
0 & 0 & 0 & -8 \delta & 0 & 0 \\
0 & 0 & 0 & 0 & -8 \delta & 0 \\
0 & 0 & 0 & 0 & 0 & -8 \delta
\end{array}\right)
$$

and $\mathbf{H}_{\mathbb{O}}$ is cubic if and only if $\delta \neq 0$. The evaluation of the invariants (5.1) on (5.2) is

$$
I_{2}(\mathbf{H})=480 \delta^{2}, \quad I_{3}(\mathbf{H})=1920 \delta^{3}, \quad I_{k}(\mathbf{H})=0 \quad \text { for } k=4 \text { to } 10 .
$$

Proposition 5.1. A minimal functional basis for $\Sigma_{[\mathbb{Q}]}$, i.e., for cubic fourth-order harmonic tensors $\mathbf{H} \in \mathbb{H}^{4}\left(\mathbb{R}^{3}\right)$, is reduced to the single rational invariant $\kappa:=I_{3} / I_{2}$.

Proof. This is a direct consequence of theorem 3.5 applied to $\mathrm{V}=\mathbb{H}^{4}\left(\mathbb{R}^{3}\right)$ and the cubic stratum $\Sigma_{[\mathbb{O}]}$ (of dimension 1). Indeed, we have $\kappa(\mathbf{H})=4 \delta \neq 0$ for all $\mathbf{H} \in \Sigma_{[\mathbb{O}]}$, and thus $I_{2}(\mathbf{H})=30 \kappa^{2}(\mathbf{H})$ and $I_{3}(\mathbf{H})=30 \kappa^{3}(\mathbf{H})$.

5.2. Transversely isotropic stratum. A fourth-order tensor $\mathbf{H} \in \mathbb{H}^{4}\left(\mathbb{R}^{3}\right)$ is at least transversely isotropic if and only if there exists a rotation $g \in \mathrm{SO}(3)$ such that $\mathbf{H}=g \star \mathbf{H}_{\mathrm{O}(2)}$, where $\mathbf{H}_{\mathrm{O}(2)}$ has the following Kelvin representation [3],

$$
\left[\mathbf{H}_{\mathrm{O}(2)}\right]=\left(\begin{array}{cccccc}
3 \delta & \delta & -4 \delta & 0 & 0 & 0 \\
\delta & 3 \delta & -4 \delta & 0 & 0 & 0 \\
-4 \delta & -4 \delta & 8 \delta & 0 & 0 & 0 \\
0 & 0 & 0 & -8 \delta & 0 & 0 \\
0 & 0 & 0 & 0 & -8 \delta & 0 \\
0 & 0 & 0 & 0 & 0 & 2 \delta
\end{array}\right)
$$

and $\mathbf{H}_{\mathrm{O}(2)}$ is transversely isotropic if and only if $\delta \neq 0$. The evaluation of the invariants (5.1) on (5.4) is

$$
\begin{array}{lll}
I_{2}=280 \delta^{2}, & I_{3}=720 \delta^{3}, & I_{4}=\frac{20000}{3} \delta^{4}, \\
I_{5}=40000 \delta^{5}, & I_{6}=\frac{2000000}{9} \delta^{6}, & I_{7}=\frac{4000000}{3} \delta^{7}, \\
I_{8}=8000000 \delta^{8}, & I_{9}=48000000 \delta^{9}, & I_{10}=800000000 \delta^{10} .
\end{array}
$$

Observe that

$$
\delta=\frac{7}{18} \frac{I_{3}}{I_{2}}=\frac{27}{250} \frac{I_{4}}{I_{3}}=\frac{1}{6} \frac{I_{5}}{I_{4}}=\frac{9}{50} \frac{I_{6}}{I_{5}}=\frac{1}{6} \frac{I_{7}}{I_{6}}=\frac{1}{6} \frac{I_{8}}{I_{7}}=\frac{1}{6} \frac{I_{9}}{I_{8}}=\frac{3}{50} \frac{I_{10}}{I_{9}},
$$

is a rational invariant. Following the same proof as for proposition 5.1, we obtain the following result.

Proposition 5.2. A minimal functional basis for $\Sigma_{[\mathrm{O}(2)]}$, i.e., for transversely isotropic fourth-order harmonic tensors $\mathbf{H} \in \mathbb{H}^{4}\left(\mathbb{R}^{3}\right)$, is reduced to the single rational invariant $\kappa:=\delta$.

5.3. Tetragonal stratum. A fourth-order tensor $\mathbf{H} \in \mathbb{H}^{4}\left(\mathbb{R}^{3}\right)$ is at least tetragonal if and only if there exists a rotation $g \in \mathrm{SO}(3)$ such that $\mathbf{H}=g \star \mathbf{H}_{\mathbb{D}_{4}}$ where $\mathbf{H}_{\mathbb{D}_{4}}$ has the following Kelvin representation,

$$
\left[\mathbf{H}_{\mathbb{D}_{4}}\right]=\left(\begin{array}{cccccc}
3 \delta-\sigma & \sigma+\delta & -4 \delta & 0 & 0 & 0 \\
\sigma+\delta & 3 \delta-\sigma & -4 \delta & 0 & 0 & 0 \\
-4 \delta & -4 \delta & 8 \delta & 0 & 0 & 0 \\
0 & 0 & 0 & -8 \delta & 0 & 0 \\
0 & 0 & 0 & 0 & -8 \delta & 0 \\
0 & 0 & 0 & 0 & 0 & 2 \sigma+2 \delta
\end{array}\right)
$$

and $\mathbf{H}_{\mathbb{D}_{4}}$ is tetragonal if and only if $\sigma \neq 0$ and $\sigma^{2}-25 \delta^{2} \neq 0$. Recall here the following bifurcation conditions [3]: (i) $\sigma=0$ implies transverse isotropy, (ii) $\sigma^{2}-25 \delta^{2}=0$ implies cubic symmetry, and (iii) 
$\sigma=0$ and $\delta=0$ imply isotropy. The evaluation of the invariants (5.1) on (5.6) is

$$
\begin{array}{lll}
I_{2}=8\left(35 \delta^{2}+\sigma^{2}\right), & I_{3}=48 \delta\left(15 \delta^{2}+\sigma^{2}\right), & I_{4}=\frac{32}{3}\left(25 \delta^{2}-\sigma^{2}\right)^{2}, \\
I_{5}=64 \delta\left(25 \delta^{2}-\sigma^{2}\right)^{2}, & I_{6}=\frac{128}{9}\left(25 \delta^{2}-\sigma^{2}\right)^{3}, & I_{7}=\frac{256}{3} \delta\left(25 \delta^{2}-\sigma^{2}\right)^{3}, \\
I_{8}=512 \delta^{2}\left(25 \delta^{2}-\sigma^{2}\right)^{3}, & I_{9}=3072 \delta^{3}\left(25 \delta^{2}-\sigma^{2}\right)^{3}, & I_{10}=2048 \delta^{2}\left(25 \delta^{2}-\sigma^{2}\right)^{4} .
\end{array}
$$

In accordance with remark B. $2, I_{4} \neq 0$ for all $\mathbf{H} \in \Sigma_{\left[\mathbb{D}_{4}\right]}$.

Proposition 5.3. A minimal functional basis for $\Sigma_{\left[\mathbb{D}_{4}\right]}$, i.e., for tetragonal fourth-order harmonic tensors $\mathbf{H} \in \mathbb{H}^{4}\left(\mathbb{R}^{3}\right)$, consists of the two rational invariants

$$
\kappa_{1}:=\frac{I_{5}}{I_{4}}, \quad \kappa_{2}:=I_{2} .
$$

Proof. For each $\mathbf{H} \in \Sigma_{\left[\mathbb{D}_{4}\right]}$, we deduce by (5.7) that

$$
\delta=\frac{1}{6} \frac{I_{5}}{I_{4}}=\frac{1}{6} \kappa_{1}, \quad \sigma^{2}=\frac{1}{8} I_{2}-35 \delta^{2}=\frac{1}{8} \kappa_{2}-\frac{35}{36} \kappa_{1}^{2} .
$$

Since each $I_{k}(2 \leq k \leq 10)$ depends only on $\delta$ and $\sigma^{2}$, we deduce that they are functions of $\kappa_{1}, \kappa_{2}$, and the proposition follows by theorem 3.5 , since $\operatorname{dim} \Sigma_{\left[\mathbb{D}_{4}\right]} / \mathrm{SO}(3)=2(6.1)$.

Remark 5.4. Neither $\left\{I_{2}, I_{3}\right\}$, nor $\left\{I_{3}, I_{4}\right\}$ are separating sets. Indeed,

- for both tetragonal tensors $(\delta=1, \sigma=\sqrt{60})$ and $(\delta=3 / 2, \sigma=\sqrt{65 / 4})$, we have $I_{2}=760$ and $I_{3}=3600$, but they have different values for $I_{4}$.

- for both tetragonal tensors $(\delta=1, \sigma=\sqrt{63})$ and $(\delta=3 / 2, \sigma=\sqrt{73 / 4})$, we have $I_{3}=3744$ and $I_{4}=46208 / 3$, but they have different values for $I_{2}$.

5.4. Trigonal stratum. A fourth-order tensor $\mathbf{H} \in \mathbb{H}^{4}\left(\mathbb{R}^{3}\right)$ is at least trigonal if and only if there exists a rotation $g \in \mathrm{SO}(3)$ such that $\mathbf{H}=g \star \mathbf{H}_{\mathbb{D}_{3}}$ where $\mathbf{H}_{\mathbb{D}_{3}}$ has the following Kelvin representation,

$$
\left[\mathbf{H}_{\mathbb{D}_{3}}\right]=\left(\begin{array}{cccccc}
3 \delta & \delta & -4 \delta & -\sqrt{2} \sigma & 0 & 0 \\
\delta & 3 \delta & -4 \delta & \sqrt{2} \sigma & 0 & 0 \\
-4 \delta & -4 \delta & 8 \delta & 0 & 0 & 0 \\
-\sqrt{2} \sigma & \sqrt{2} \sigma & 0 & -8 \delta & 0 & 0 \\
0 & 0 & 0 & 0 & -8 \delta & -2 \sigma \\
0 & 0 & 0 & 0 & -2 \sigma & 2 \delta
\end{array}\right)
$$

and $\mathbf{H}_{\mathbb{D}_{3}}$ is trigonal if and only if $\sigma \neq 0$ and $\sigma^{2}-50 \delta^{2} \neq 0$. Recall also the bifurcation conditions [3]: $(i)$ $\sigma=0$ implies transverse isotropy, $(i i) \sigma^{2}-50 \delta^{2}=0$ implies cubic symmetry, and (iii) $\sigma=0$ and $\delta=0$ imply isotropy. The evaluation of the invariants (5.1) on (5.9) is

$$
\begin{array}{lll}
I_{2}=8\left(35 \delta^{2}+2 \sigma^{2}\right), & I_{3}=144 \delta\left(5 \delta^{2}-\sigma^{2}\right), & I_{4}=\frac{8}{3}\left(50 \delta^{2}-\sigma^{2}\right)^{2}, \\
I_{5}=16 \delta\left(50 \delta^{2}-\sigma^{2}\right)^{2}, & I_{6}=\frac{16}{9}\left(50 \delta^{2}-\sigma^{2}\right)^{3}, & I_{7}=\frac{32}{3} \delta\left(50 \delta^{2}-\sigma^{2}\right)^{3}, \\
I_{8}=64 \delta^{2}\left(50 \delta^{2}-\sigma^{2}\right)^{3}, & I_{9}=384 \delta^{3}\left(50 \delta^{2}-\sigma^{2}\right)^{3} & I_{10}=128 \delta^{2}\left(50 \delta^{2}-\sigma^{2}\right)^{4} .
\end{array}
$$

As for the tetragonal case, we have $I_{4} \neq 0$ for all $\mathbf{H} \in \Sigma_{\left[\mathbb{D}_{3}\right]}$. Now, following the same proof as the one of proposition 5.3, we get:

Proposition 5.5. A minimal functional basis for $\Sigma_{\left[\mathbb{D}_{3}\right]}$, i.e., for trigonal harmonic fourth-order tensors $\mathbf{H} \in \mathbb{H}^{4}\left(\mathbb{R}^{3}\right)$, consists of the two rational invariants

$$
\kappa_{1}:=\frac{I_{5}}{I_{4}}, \quad \kappa_{2}:=I_{2} .
$$

Remark 5.6. Neither $\left\{I_{2}, I_{3}\right\}$ nor $\left\{I_{3}, I_{4}\right\}$ are separating sets. Indeed,

- for both trigonal tensors $(\delta=1, \sigma=\sqrt{715 / 8})$ and $(\delta=3 / 2, \sigma=\sqrt{135 / 2})$, we have $I_{2}=1710$ and $I_{3}=-12150$, but they have different values for $I_{4}$.

- for both trigonal tensors $(\delta=1, \sigma=\sqrt{371 / 4})$ and $(\delta=3 / 2, \sigma=\sqrt{279 / 4})$, we have $I_{3}=-12636$ and $I_{4}=9747 / 2$, but they have different values for $I_{2}$.

We point out here that each proposed minimal functional basis concerns an exact symmetry stratum. The proposed functional basis $\left\{\kappa_{1}=I_{5} / I_{4}, \kappa_{2}=I_{2}\right\}$ happens to be identical for the tetragonal and trigonal strata. A natural question then arises: does this set remain a functional basis for the union of strata $\Sigma_{\left[\mathbb{D}_{3}\right]} \cup \Sigma_{\left[\mathbb{D}_{4}\right]}$ ? The answer is no as detailed in the following remark. 
Remark 5.7. By proposition 5.3, two tetragonal harmonic fourth-order tensors having the same values for $\kappa_{1}$ and $\kappa_{2}$ are indeed in the same orbit (as a functional basis is a separating set). The same holds, by proposition 5.5, if one considers two trigonal harmonic fourth-order tensors having the same values for $\kappa_{1}$ and $\kappa_{2}$. There exists, however, trigonal tensors that have the same value for $\kappa_{1}$ and $\kappa_{2}$ as some tetragonal tensors. Since, they are not on the same orbit as they do not belong to the same symmetry class, and the set $\left\{\kappa_{1}, \kappa_{2}\right\}$ is not a functional basis for $\Sigma_{\left[\mathbb{D}_{3}\right]} \cup \Sigma_{\left[\mathbb{D}_{4}\right]}$.

5.5. Orthotropic stratum. A fourth-order tensor $\mathbf{H} \in \mathbb{H}^{4}\left(\mathbb{R}^{3}\right)$ is at least orthotropic if and only if there exists a rotation $g \in \mathrm{SO}(3)$ such that $\mathbf{H}=g \star \mathbf{H}_{\mathbb{D}_{2}}$ where $\mathbf{H}_{\mathbb{D}_{2}}$ has the following Kelvin representation [28],

$$
\left[\mathbf{H}_{\mathbb{D}_{2}}\right]=\left(\begin{array}{cccccc}
\lambda_{2}+\lambda_{3} & -\lambda_{3} & -\lambda_{2} & 0 & 0 & 0 \\
-\lambda_{3} & \lambda_{3}+\lambda_{1} & -\lambda_{1} & 0 & 0 & 0 \\
-\lambda_{2} & -\lambda_{1} & \lambda_{1}+\lambda_{2} & 0 & 0 & 0 \\
0 & 0 & 0 & -2 \lambda_{1} & 0 & 0 \\
0 & 0 & 0 & 0 & -2 \lambda_{2} & 0 \\
0 & 0 & 0 & 0 & 0 & -2 \lambda_{3}
\end{array}\right),
$$

and $\mathbf{H}_{\mathbb{D}_{2}}$ is orthotropic if and only if $\lambda_{1}, \lambda_{2}, \lambda_{3}$ are all distinct. In fact, setting

$$
\Delta:=\left(\lambda_{1}-\lambda_{2}\right)\left(\lambda_{2}-\lambda_{3}\right)\left(\lambda_{1}-\lambda_{3}\right),
$$

we have by direct evaluation of the invariant $\left\|\operatorname{tr}\left(\mathbf{H} \times \mathbf{d}_{2}\right)\right\|^{2}$ on (5.12):

$$
\left\|\operatorname{tr}\left(\mathbf{H} \times \mathbf{d}_{2}\right)\right\|^{2}=\frac{6}{25} \Delta^{2} .
$$

The evaluation of the integrity basis $\left\{I_{2}, \ldots, I_{10}\right\}$ of $\mathbf{H}$ on (5.12) can be expressed polynomially using the elementary symmetric functions [3, section 5.5]

$$
\sigma_{1}:=\lambda_{1}+\lambda_{2}+\lambda_{3}, \quad \sigma_{2}:=\lambda_{1} \lambda_{2}+\lambda_{2} \lambda_{3}+\lambda_{2} \lambda_{3}, \quad \sigma_{3}:=\lambda_{1} \lambda_{2} \lambda_{3} .
$$

Conversely, the $\sigma_{i}$ can be expressed rationally in the $I_{k}$.

Proposition 5.8. A minimal functional basis for $\Sigma_{\left[\mathbb{D}_{2}\right]}$, i.e., for orthotropic harmonic fourth-order tensors $\mathbf{H} \in \mathbb{H}^{4}\left(\mathbb{R}^{3}\right)$, consists of the three rational invariants

$$
\begin{aligned}
\sigma_{1} & :=\frac{1}{96} \frac{6 I_{7}+3 I_{3} I_{4}-2 I_{2} I_{5}}{\Delta^{2}}, \\
\sigma_{2} & :=\frac{4}{7} \sigma_{1}^{2}-\frac{1}{14} I_{2}, \\
\sigma_{3} & :=\frac{1}{7} \sigma_{1}^{3}-\frac{1}{56} \sigma_{1} I_{2}-\frac{1}{24} I_{3},
\end{aligned}
$$

where $\Delta^{2}=\frac{1}{1296}\left(2 I_{2}{ }^{3}-60 I_{3}{ }^{2}-9 I_{2} I_{4}+18 I_{6}\right) \neq 0$,

Proof. For each $\mathbf{H} \in \Sigma_{\left[\mathbb{D}_{2}\right]}$, we can write $\mathbf{H}=g \star \mathbf{H}_{\mathbb{D}_{2}}$ where $\mathbf{H}_{\mathbb{D}_{2}}$ is given by (5.12). Now, a direct evaluation on the normal form (5.12) leads to

$$
6 I_{7}+3 I_{3} I_{4}-2 I_{2} I_{5}=96 \sigma_{1} \Delta^{2}, \quad 2 I_{2}^{3}-60 I_{3}^{2}-9 I_{2} I_{4}+18 I_{6}=1296 \Delta^{2} .
$$

Hence, we obtain the first equation of (5.14), while the others are obtained in the same way. Finally, each invariant $I_{2}, \ldots, I_{10}$ is a polynomial function of $\sigma_{1}, \sigma_{2}, \sigma_{3}[3]$, and, since $\operatorname{dim} \Sigma_{\left[\mathbb{D}_{2}\right]} / \mathrm{SO}(3)=3$, the conclusion follows by theorem 3.5.

\section{Functional Bases on symmetry strata of Elasticity tensors}

We finally address the problem of the determination of minimal functional bases for the symmetry strata of the elasticity tensor (except for the orthotropic $\Sigma_{\left[\mathbb{D}_{2}\right]}$, the monoclinic $\Sigma_{\left[\mathbb{Z}_{2}\right]}$ and the triclinic $\Sigma_{[\mathbb{1}]}$ strata, which will be investigated in a future work). The isotropic case is trivial, a minimal functional basis for the isotropic stratum $\Sigma_{[\mathrm{SO}(3)]}$ consists of the two Lamé coefficients. The cubic case is straightforward and treated in section 6.1. In order to derive our results for the trigonal $\Sigma_{\left[\mathbb{D}_{3}\right]}$, tetragonal $\Sigma_{\left[\mathbb{D}_{4}\right]}$ and transversely isotropic $\Sigma_{[\mathrm{O}(2)]}$ strata, we shall define in section 6.2 a non vanishing second-order covariant $\mathbf{t}=\mathbf{t}(\mathbf{E})$ of $\mathbf{E}$.

We recall the dimensions of the eight orbit spaces $\Sigma_{[H]} / \mathrm{SO}(3)$ (see [3]),

$$
\begin{array}{lll}
\operatorname{dim}\left(\Sigma_{[\mathbb{1}]} / \mathrm{SO}(3)\right)=18, & \operatorname{dim}\left(\Sigma_{\left[\mathbb{Z}_{2}\right]} / \mathrm{SO}(3)\right)=12, & \operatorname{dim}\left(\Sigma_{\left[\mathbb{D}_{2}\right]} / \mathrm{SO}(3)\right)=9, \\
\operatorname{dim}\left(\Sigma_{\left[\mathbb{D}_{4}\right]} / \mathrm{SO}(3)\right)=6, & \operatorname{dim}\left(\Sigma_{\left[\mathbb{D}_{3}\right]} / \mathrm{SO}(3)\right)=6, & \operatorname{dim}\left(\Sigma_{[\mathrm{O}(2)]} / \mathrm{SO}(3)\right)=5, \\
\operatorname{dim}\left(\Sigma_{[\mathbb{O}]} / \mathrm{SO}(3)\right)=3, & \operatorname{dim}\left(\Sigma_{[\mathrm{SO}(3)]} / \mathrm{SO}(3)\right)=2 . &
\end{array}
$$


6.1. Elasticity cubic stratum. By theorem 2.1, an elasticity tensor

$$
\mathbf{E}=\left(\operatorname{tr} \mathbf{d}, \operatorname{tr} \mathbf{v}, \mathbf{d}^{\prime}, \mathbf{v}^{\prime}, \mathbf{H}\right) \in \mathbb{E l a}
$$

is cubic if and only if $\mathbf{d}^{\prime}=\mathbf{v}^{\prime}=\mathbf{d}_{2}^{\prime}=0$ and $I_{2}(\mathbf{H})=\operatorname{tr} \mathbf{d}_{2} \neq 0$ (meaning that $\mathbf{H} \in \mathbb{H}^{4}\left(\mathbb{R}^{3}\right)$ is cubic). Now, by proposition 5.1 and $\operatorname{since} \operatorname{dim}\left(\Sigma_{[\mathbb{0}]} / \mathrm{SO}(3)\right)=3$, we have the following result.

Theorem 6.1. Let $\mathbf{E}=(\operatorname{tr} \mathbf{d}, \operatorname{tr} \mathbf{v}, 0,0, \mathbf{H})$ be a cubic elasticity tensor. A minimal functional basis for $\Sigma_{[\mathbb{O}]}$ consists of the three rational invariants

$$
\kappa_{1}:=\operatorname{tr} \mathbf{d}, \quad \kappa_{2}:=\operatorname{tr} \mathbf{v}, \quad \kappa_{3}:=\frac{I_{3}}{I_{2}} .
$$

6.2. A transversely isotropic second-order covariant. The goal, here, is to build a symmetric second-order covariant of $\mathbf{E} \in \mathbb{E}$ la which is strictly transversely isotropic for all trigonal, tetragonal and transversely isotropic tensors. Observe that each symmetric second-order covariant, $\mathbf{t}(\mathbf{E})$, is necessarily at least transversely isotropic since it inherits the symmetries of $\mathbf{E}$ and since a second-order symmetric tensor can only be either orthotropic (three distinct eigenvalues), transversely isotropic (two distinct eigenvalues) or isotropic (only one eigenvalue). It is however not obvious to find such a covariant which remains strictly transversely isotropic for all

$$
\mathbf{E} \in \Sigma_{\left[\mathbb{D}_{3}\right]} \cup \Sigma_{\left[\mathbb{D}_{4}\right]} \cup \Sigma_{[\mathrm{O}(2)]} .
$$

To build such a covariant, we use corollary 2.2, which forbids $\left(\mathbf{d}^{\prime}, \mathbf{v}^{\prime}, \mathbf{d}_{2}^{\prime}\right)$ to be isotropic, and denote by $\langle\boldsymbol{n}\rangle$ the direction of transverse isotropy of the triplet $\left(\mathbf{d}^{\prime}, \mathbf{v}^{\prime}, \mathbf{d}_{2}^{\prime}\right)$. By proposition 4.4 , with $\|\boldsymbol{n}\|=1$, $\left\|(\boldsymbol{n} \otimes \boldsymbol{n})^{\prime}\right\|=\sqrt{\frac{2}{3}}$, we get thus

$$
\mathbf{d}^{\prime}= \pm \sqrt{\frac{3}{2}}\left\|\mathbf{d}^{\prime}\right\|(\boldsymbol{n} \otimes \boldsymbol{n})^{\prime}, \quad \mathbf{v}^{\prime}= \pm \sqrt{\frac{3}{2}}\left\|\mathbf{v}^{\prime}\right\|(\boldsymbol{n} \otimes \boldsymbol{n})^{\prime}, \quad \mathbf{d}_{2}^{\prime}= \pm \sqrt{\frac{3}{2}}\left\|\mathbf{d}_{2}^{\prime}\right\|(\boldsymbol{n} \otimes \boldsymbol{n})^{\prime},
$$

and

$$
\left\|\mathbf{d}^{\prime}\right\|^{2} \mathbf{d}^{\prime 2}+\left\|\mathbf{v}^{\prime}\right\|^{2} \mathbf{v}^{\prime 2}+\mathbf{d}_{2}^{\prime 2}=\frac{3}{2}\left(\left\|\mathbf{d}^{\prime}\right\|^{4}+\left\|\mathbf{v}^{\prime}\right\|^{4}+\left\|\mathbf{d}_{2}^{\prime}\right\|^{2}\right)(\boldsymbol{n} \otimes \boldsymbol{n})^{\prime 2} .
$$

The property $\left((\boldsymbol{n} \otimes \boldsymbol{n})^{\prime 2}\right)^{\prime}=\frac{1}{3}(\boldsymbol{n} \otimes \boldsymbol{n})^{\prime}$ leads to

$$
\left(\left\|\mathbf{d}^{\prime}\right\|^{2} \mathbf{d}^{\prime 2}+\left\|\mathbf{v}^{\prime}\right\|^{2} \mathbf{v}^{\prime 2}+\mathbf{d}_{2}^{\prime 2}\right)^{\prime}=\frac{1}{2}\left(\left\|\mathbf{d}^{\prime}\right\|^{4}+\left\|\mathbf{v}^{\prime}\right\|^{4}+\left\|\mathbf{d}_{2}^{\prime}\right\|^{2}\right)(\boldsymbol{n} \otimes \boldsymbol{n})^{\prime} \neq 0
$$

as $\left\|\mathbf{d}^{\prime}\right\|^{4}+\left\|\mathbf{v}^{\prime}\right\|^{4}+\left\|\mathbf{d}_{2}^{\prime}\right\|^{2} \neq 0$ over the entire union of strata $\Sigma_{[\mathrm{O}(2)]} \cup \Sigma_{\left[\mathbb{D}_{3}\right]} \cup \Sigma_{\left[\mathbb{D}_{4}\right]}$.

Therefore, this allows us to define the deviatoric second-order rational covariant

$$
\mathbf{t}:=2 \frac{\left(\left\|\mathbf{d}^{\prime}\right\|^{2} \mathbf{d}^{\prime 2}+\left\|\mathbf{v}^{\prime}\right\|^{2} \mathbf{v}^{\prime 2}+\mathbf{d}_{2}^{\prime 2}\right)^{\prime}}{\left\|\mathbf{d}^{\prime}\right\|^{4}+\left\|\mathbf{v}^{\prime}\right\|^{4}+\left\|\mathbf{d}_{2}^{\prime}\right\|^{2}} \neq 0
$$

for every elasticity tensor which is either trigonal, tetragonal or transversely isotropic. It is normalized in such a way that

$$
\mathbf{t}=(\boldsymbol{n} \otimes \boldsymbol{n})^{\prime}, \quad\|\boldsymbol{n}\|=1, \quad\|\mathbf{t}\|=\sqrt{\frac{2}{3}},
$$

and thus ( since $\mathbf{t}=\mathbf{t}^{\prime}$ )

$$
\mathbf{d}^{\prime}=\frac{3}{2}(\mathbf{d}: \mathbf{t}) \mathbf{t}, \quad \mathbf{v}^{\prime}=\frac{3}{2}(\mathbf{v}: \mathbf{t}) \mathbf{t}, \quad \mathbf{d}_{2}^{\prime}=\frac{3}{2}\left(\mathbf{d}_{2}: \mathbf{t}\right) \mathbf{t} .
$$

6.3. Elasticity transversely isotropic stratum. By corollary 2.2, if $\mathbf{E}$ is transversely isotropic, then, the triplet $\left(\mathbf{d}^{\prime}, \mathbf{v}^{\prime}, \mathbf{d}_{2}^{\prime}\right)$ is transversely isotropic, and thus

$$
\left\|\mathbf{d}^{\prime}\right\|^{4}+\left\|\mathbf{v}^{\prime}\right\|^{4}+\left\|\mathbf{d}_{2}^{\prime}\right\|^{2} \neq 0 .
$$

Theorem 6.2. Let $\mathbf{E}=\left(\operatorname{tr} \mathbf{d}, \operatorname{tr} \mathbf{v}, \mathbf{d}^{\prime}, \mathbf{v}^{\prime}, \mathbf{H}\right) \in \Sigma_{[\mathrm{O}(2)]}$ be a transversely isotropic elasticity tensor and

$$
\mathbf{t}=2 \frac{\left(\left\|\mathbf{d}^{\prime}\right\|^{2} \mathbf{d}^{\prime 2}+\left\|\mathbf{v}^{\prime}\right\|^{2} \mathbf{v}^{\prime 2}+\mathbf{d}_{2}^{\prime 2}\right)^{\prime}}{\left\|\mathbf{d}^{\prime}\right\|^{4}+\left\|\mathbf{v}^{\prime}\right\|^{4}+\left\|\mathbf{d}_{2}^{\prime}\right\|^{2}} \in \mathbb{H}^{2}\left(\mathbb{R}^{3}\right)
$$

A minimal functional basis for $\Sigma_{[\mathrm{O}(2)]}$ consists of the five rational invariants

$$
\kappa_{1}:=\operatorname{tr} \mathbf{d}, \quad \kappa_{2}:=\operatorname{tr} \mathbf{v}, \quad \kappa_{3}:=\mathbf{d}: \mathbf{t}, \quad \kappa_{4}:=\mathbf{v}: \mathbf{t}, \quad \kappa_{5}:=\mathbf{t}: \mathbf{H}: \mathbf{t} .
$$


Proof. Let $\mathbf{E}$ and $\overline{\mathbf{E}}$ be two transversely isotropic elasticity tensors with the same invariants $\kappa_{1}, \ldots, \kappa_{5}$. We have to show that there exists $g \in \mathrm{SO}(3)$, such that

$$
g \star \mathbf{d}^{\prime}=\overline{\mathbf{d}^{\prime}}, \quad g \star \mathbf{v}^{\prime}=\overline{\mathbf{v}^{\prime}}, \quad g \star \mathbf{H}=\overline{\mathbf{H}} .
$$

Now, $\mathbf{t}(\mathbf{E})$ being the covariant defined by (6.5), we can write (see section 6.2)

$$
\mathbf{t}=(\boldsymbol{n} \otimes \boldsymbol{n})^{\prime}, \quad \overline{\mathbf{t}}=(\overline{\boldsymbol{n}} \otimes \overline{\boldsymbol{n}})^{\prime},
$$

where $\boldsymbol{n}$ and $\overline{\boldsymbol{n}}$ are two unit vectors. Choose a rotation $g \in \mathrm{SO}(3)$ such that $g \boldsymbol{n}=\overline{\boldsymbol{n}}$. Then, we get $g \star \mathbf{t}=\overline{\mathbf{t}}$, and by (6.4) and proposition 4.4

$$
\mathbf{d}=\frac{\kappa_{1}}{3} \mathbf{q}+\frac{3}{2}(\mathbf{d}: \mathbf{t}) \mathbf{t}=\frac{\kappa_{1}}{3} \mathbf{q}+\frac{3}{2} \kappa_{2} \mathbf{t} \Longrightarrow g \star \mathbf{d}=\frac{\kappa_{1}}{3} \mathbf{q}+\frac{3}{2} \kappa_{2} g \star \mathbf{t}=\overline{\mathbf{d}} .
$$

The argumentation is the same for $\mathbf{v}$ and $\overline{\mathbf{v}}$. Finally, using the reconstruction formula (C.2), we have

$$
\mathbf{H}=\frac{35}{8}(\mathbf{t}: \mathbf{H}: \mathbf{t}) \mathbf{t} * \mathbf{t}=\frac{35}{8} \kappa_{5} \mathbf{t} * \mathbf{t} \Longrightarrow g \star \mathbf{H}=\frac{35}{8} \kappa_{5}(g \star \mathbf{t}) *(g \star \mathbf{t})=\overline{\mathbf{H}},
$$

where the harmonic square $\mathbf{t} * \mathbf{t}$ is the fourth order harmonic part of $\mathbf{t} \odot \mathbf{t}=(\mathbf{t} \otimes \mathbf{t})^{s}$,

$$
\mathbf{t} * \mathbf{t}:=\mathbf{t} \odot \mathbf{t}-\frac{4}{7} \mathbf{q} \odot \mathbf{t}^{2}+\frac{2}{35}\|\mathbf{t}\|^{2} \mathbf{q} \odot \mathbf{q},
$$

such as $g \star(\mathbf{t} * \mathbf{t})=(g \star \mathbf{t}) *(g \star \mathbf{t})$. This achieves the proof that $\left\{\kappa_{1}, \ldots, \kappa_{5}\right\}$ is a functional basis for $\Sigma_{[\mathrm{O}(2)]}$ and the minimality follows by remark 3.4 , since $\operatorname{dim}\left(\Sigma_{[\mathrm{O}(2)]} / \mathrm{SO}(3)\right)=5$.

6.4. Elasticity tetragonal stratum. Given a tetragonal elasticity tensor

$$
\mathbf{E}=\left(\operatorname{tr} \mathbf{d}, \operatorname{tr} \mathbf{v}, \mathbf{d}^{\prime}, \mathbf{v}^{\prime}, \mathbf{H}\right) \in \Sigma_{\left[\mathbb{D}_{4}\right]},
$$

the triplet $\left(\mathbf{d}^{\prime}, \mathbf{v}^{\prime}, \mathbf{d}_{2}^{\prime}\right)$ is transversely isotropic (by corollary 2.2) and $\mathbf{H} \in \mathbb{H}^{4}\left(\mathbb{R}^{3}\right)$ is either cubic or tetragonal (it is neither isotropic, nor transversely isotropic [17, 29]).

Theorem 6.3. Let $\mathbf{E}=\left(\operatorname{tr} \mathbf{d}, \operatorname{tr} \mathbf{v}, \mathbf{d}^{\prime}, \mathbf{v}^{\prime}, \mathbf{H}\right)$ be a tetragonal elasticity tensor and

$$
\mathbf{t}=2 \frac{\left(\left\|\mathbf{d}^{\prime}\right\|^{2} \mathbf{d}^{\prime 2}+\left\|\mathbf{v}^{\prime}\right\|^{2} \mathbf{v}^{\prime 2}+\mathbf{d}_{2}^{\prime 2}\right)^{\prime}}{\left\|\mathbf{d}^{\prime}\right\|^{4}+\left\|\mathbf{v}^{\prime}\right\|^{4}+\left\|\mathbf{d}_{2}^{\prime}\right\|^{2}} .
$$

A minimal functional basis for $\Sigma_{\left[\mathbb{D}_{4}\right]}$ consists of the six rational invariants

$$
\begin{array}{lll}
\kappa_{1}:=\operatorname{tr} \mathbf{d}, & \kappa_{2}:=\operatorname{tr} \mathbf{v}, & \kappa_{3}:=\mathbf{d}: \mathbf{t}, \\
\kappa_{4}:=\mathbf{v}: \mathbf{t}, & \kappa_{5}:=\mathbf{t}: \mathbf{H}: \mathbf{t}, & \kappa_{6}:=I_{2} .
\end{array}
$$

Remark 6.4. In this set, $\kappa_{6}=I_{2}=\operatorname{tr} \mathbf{d}_{2}$ can be replaced by $I_{3}=\operatorname{tr} \mathbf{d}_{3}$ (by lemma D.1).

Proof. Let $\mathbf{E}$ and $\overline{\mathbf{E}}$ be two tetragonal elasticity tensors. Then, the pairs $(\mathbf{H}, \mathbf{t})$ and $(\overline{\mathbf{H}}, \overline{\mathbf{t}})$ are necessarily both tetragonal, since they have the same respective symmetry as $\left(\mathbf{d}^{\prime}, \mathbf{v}^{\prime}, \mathbf{H}\right)$ and $\left(\overline{\mathbf{d}^{\prime}}, \overline{\mathbf{v}^{\prime}}, \overline{\mathbf{H}}\right)$. If they have the same invariants $\kappa_{1}, \ldots, \kappa_{6}$, then,

$$
\kappa_{5}=\mathbf{t}: \mathbf{H}: \mathbf{t}=\overline{\mathbf{t}}: \overline{\mathbf{H}}: \overline{\mathbf{t}}, \quad \text { and } \quad \kappa_{6}=I_{2}(\mathbf{H})=I_{2}(\overline{\mathbf{H}}),
$$

and thus, by lemma D.1, $I_{k}(\mathbf{H})=I_{k}(\overline{\mathbf{H}})$ for $2 \leq k \leq 10$. Hence, there exists $g \in \operatorname{SO}(3)$ such that $g \star \mathbf{H}=\overline{\mathbf{H}}$. Now, two cases can happen.

(1) $\mathbf{H}$ is tetragonal and has thus the same symmetry group as the pair $(\mathbf{H}, \mathbf{t})$ (the same holds for $\overline{\mathbf{H}}$ and $(\overline{\mathbf{H}}, \overline{\mathbf{t}}))$. In that case, let $\langle\boldsymbol{n}\rangle$ be the principal axis of symmetry group of $\mathbf{H}$, and $\langle\overline{\boldsymbol{n}}\rangle$, the one for $\overline{\mathbf{H}}$. Then, $g \boldsymbol{n}= \pm \overline{\boldsymbol{n}}$ and thus $g \star \mathbf{t}=\overline{\mathbf{t}}$.

(2) $\mathbf{H}$ is cubic. Then, the principal axis $\langle\boldsymbol{n}\rangle$ of the tetragonal pair $(\mathbf{H}, \mathbf{t})$ is necessarily one of the three principal axes of the cubic tensor $\mathbf{H}$ (and similarly for the pair $(\overline{\mathbf{H}}, \overline{\mathbf{t}})$ ). Since $g$ sends each principal axis of $\mathbf{H}$ onto a principal axis of $\overline{\mathbf{H}}$, it is possible to change $g$ such that $g \boldsymbol{n}=\overline{\boldsymbol{n}}$, and thus that $g \star \mathbf{t}=\overline{\mathbf{t}}$ (keeping $g \star \mathbf{H}=\overline{\mathbf{H}}$ ), by replacing $g$ by $g h$, where $h$ belongs to the symmetry group of $\mathbf{H}$ (see [28, Lemma 8.9] for details).

In both cases, we conclude as in the proof of theorem 6.2 , and the minimality follows since $\operatorname{dim}\left(\Sigma_{\left[\mathbb{D}_{4}\right]} / \mathrm{SO}(3)\right)=$ 6 . 
6.5. Elasticity trigonal stratum. Given a trigonal elasticity tensor

$$
\mathbf{E}=\left(\operatorname{tr} \mathbf{d}, \operatorname{tr} \mathbf{v}, \mathbf{d}^{\prime}, \mathbf{v}^{\prime}, \mathbf{H}\right) \in \Sigma_{\left[\mathbb{D}_{3}\right]},
$$

the triplet $\left(\mathbf{d}^{\prime}, \mathbf{v}^{\prime}, \mathbf{d}_{2}^{\prime}\right)$ is transversely isotropic (by corollary 2.2) and $\mathbf{H} \in \mathbb{H}^{4}\left(\mathbb{R}^{3}\right)$ is either cubic or trigonal (it is neither isotropic, nor transversely isotropic $[17,29]$ ). The proof of the following result is obtained in the same way as in the tetragonal case.

Theorem 6.5. Let $\mathbf{E}=\left(\operatorname{tr} \mathbf{d}, \operatorname{tr} \mathbf{v}, \mathbf{d}^{\prime}, \mathbf{v}^{\prime}, \mathbf{H}\right)$ be a trigonal elasticity tensor and

$$
\mathbf{t}=2 \frac{\left(\left\|\mathbf{d}^{\prime}\right\|^{2} \mathbf{d}^{\prime 2}+\left\|\mathbf{v}^{\prime}\right\|^{2} \mathbf{v}^{\prime 2}+\mathbf{d}_{2}^{\prime 2}\right)^{\prime}}{\left\|\mathbf{d}^{\prime}\right\|^{4}+\left\|\mathbf{v}^{\prime}\right\|^{4}+\left\|\mathbf{d}_{2}^{\prime}\right\|^{2}} .
$$

A minimal functional basis for $\Sigma_{\left[\mathbb{D}_{3}\right]}$ consists of the six rational invariants

$$
\begin{array}{lll}
\kappa_{1}:=\operatorname{tr} \mathbf{d}, & \kappa_{2}:=\operatorname{tr} \mathbf{v}, & \kappa_{3}:=\mathbf{d}: \mathbf{t}, \\
\kappa_{4}:=\mathbf{v}: \mathbf{t}, & \kappa_{5}:=\mathbf{t}: \mathbf{H}: \mathbf{t}, & \kappa_{6}=I_{2} .
\end{array}
$$

Remark 6.6. In this set, the invariant $\kappa_{6}=I_{2}=\operatorname{tr} \mathbf{d}_{2}$ can be changed into $I_{3}=\operatorname{tr} \mathbf{d}_{3}$ (by lemma D.1).

6.6. The special case of fourth-order harmonic tensors. The theorems provided in section 6 apply, of course, to fourth-order harmonic tensors $\mathbf{H} \in \mathbb{H}^{4}\left(\mathbb{R}^{3}\right) \subset \mathbb{E}$ la (as the special case $\mathbf{d}=\mathbf{v}=0$ ). In the cubic case, the functional basis defined by the single invariant $I_{3} / I_{2}$ in proposition 5.1 is trivially recovered. In the transversely isotropic, tetragonal and trigonal cases, the functional bases provided in propositions 5.2, 5.3 and 5.5 are recovered as special cases of theorems 6.2, 6.3 and 6.5, thanks to the equalities $\mathbf{H}: \mathbf{d}_{2}=2 \mathbf{d}_{2}^{\prime}\left[28\right.$, Remark 5.2], $\mathbf{d}_{2}^{\prime}= \pm \sqrt{\frac{3}{2}}\left\|\mathbf{d}_{2}^{\prime}\right\| \mathbf{t} \neq 0$ (see section 6.2) and the definitions (5.1), so that

$$
\mathbf{t}: \mathbf{H}: \mathbf{t}=\frac{2}{3} \frac{\mathbf{d}_{2}^{\prime}: \mathbf{H}: \mathbf{d}_{2}^{\prime}}{\left\|\mathbf{d}_{2}^{\prime}\right\|^{2}}=\frac{4}{3} \frac{\mathbf{d}_{2}^{\prime}: \mathbf{d}_{3}^{\prime}}{I_{4}}=\frac{4 I_{5}}{3 I_{4}} .
$$

The equality $\mathbf{t}: \mathbf{H}: \mathbf{t}=4 I_{5} / 3 I_{4}$ is valid for any transversely isotropic, tetragonal or trigonal pair $(\mathbf{H}, \mathbf{t})$ with $\left\|\mathbf{t}^{\prime}\right\|=\sqrt{2 / 3}$.

\section{A POLYNOMIAL FUNCTIONAL BASIS FOR ELASTICITY TENSORS AT LEAST TETRAGONAL OR TRIGONAL}

By remark 5.7, each functional basis obtained in the previous section is a priori valid for one and only one elasticity symmetry stratum, among the cubic, the transversely isotropic, the tetragonal and the trigonal ones ${ }^{2}$. Consider now the union of strata

$$
X:=\Sigma_{[\mathrm{SO}(3)]} \cup \Sigma_{[\mathbb{O}]} \cup \Sigma_{[\mathrm{O}(2)]} \cup \Sigma_{\left[\mathbb{D}_{3}\right]} \cup \Sigma_{\left[\mathbb{D}_{4}\right]} \subset \mathbb{E l a} .
$$

Of course, for each of these strata, the set of numerators and denominators of the rational invariants involved in their respective rational separating set obtained, constitutes a separating set of polynomial invariants for each of them. But the union of these sets is not separating for $X$ (see remark 5.7). The question is thus whether one can merge and complete these separating sets in order to build a polynomial separating set, and hence a polynomial functional basis, valid for any elasticity tensors $\mathbf{E}$ at least tetragonal or trigonal, i.e., for $\mathrm{X}$. A positive response is provided by the following result (see Appendix E for a proof).

Theorem 7.1. Let $\mathbf{E}=\left(\operatorname{tr} \mathbf{d}, \operatorname{tr} \mathbf{v}, \mathbf{d}^{\prime}, \mathbf{v}^{\prime}, \mathbf{H}\right)$ be an elasticity tensor and

$$
\begin{array}{llll}
K_{1}:=\operatorname{tr} \mathbf{d}, & L_{1}:=\operatorname{tr} \mathbf{v}, & I_{3}:=\operatorname{tr} \mathbf{d}_{3}, & K_{4}:=\left\|\mathbf{d}^{\prime}\right\|^{4}+\left\|\mathbf{v}^{\prime}\right\|^{4}+\left\|\mathbf{d}_{2}^{\prime}\right\|^{2}, \\
K_{5}:=\mathbf{d}: \mathbf{k}_{4}, & L_{5}:=\mathbf{v}: \mathbf{k}_{4}, & K_{9}:=\mathbf{k}_{4}: \mathbf{H}: \mathbf{k}_{4}, & K_{10}:=\left\|\operatorname{tr}\left(\mathbf{H} \times \mathbf{k}_{4}\right)\right\|^{2} .
\end{array}
$$

where $\mathbf{k}_{4}:=\left(\left\|\mathbf{d}^{\prime}\right\|^{2} \mathbf{d}^{\prime 2}+\left\|\mathbf{v}^{\prime}\right\|^{2} \mathbf{v}^{\prime 2}+\mathbf{d}_{2}^{\prime 2}\right)^{\prime}$.

(1) A minimal functional basis for $\Sigma_{[\mathrm{SO}(3)]} \cup \Sigma_{[\mathbb{O}]} \cup \Sigma_{[\mathrm{O}(2)]} \cup \Sigma_{\left[\mathbb{D}_{4}\right]}$ (i.e., at least tetragonal elasticity tensors) consists of the seven polynomial invariants $K_{1}, L_{1}, I_{3}, K_{4}, K_{5}, L_{5}$ and $K_{9}$.

(2) A minimal functional basis for $\Sigma_{[\mathrm{SO}(3)]} \cup \Sigma_{[\mathbb{O}]} \cup \Sigma_{[\mathrm{O}(2)]} \cup \Sigma_{\left[\mathbb{D}_{3}\right]}$ (i.e., at least trigonal elasticity tensors) consists of the seven polynomial invariants $K_{1}, L_{1}, I_{3}, K_{4}, K_{5}, L_{5}$ and $K_{9}$.

(3) A minimal functional basis for $\mathrm{X}$ (i.e., at least tetragonal or trigonal elasticity tensors) consists of the eight polynomial invariants $K_{1}, L_{1}, I_{3}, K_{4}, K_{5}, L_{5}, K_{9}$ and $K_{10}$.

\footnotetext{
${ }^{2}$ The two invariants $\operatorname{tr} \mathbf{d}, \operatorname{tr} \mathbf{v}$ constitute a minimal functional basis for the isotropic stratum $\Sigma_{[\mathrm{SO}(3)]}$.
} 


\section{Conclusion}

We have summarized the mathematical material that allows to define the notion of minimal functional basis, not only on a whole vector space (such as $\mathbb{E}$ la) but also on its symmetry strata $\Sigma_{[H]}$. Restricting the concept of functional basis to the class of continuous functions, we have been able to define a lower bound for the cardinality of such a basis for a stratum $\Sigma_{[H]}$ (namely, $\operatorname{dim} \Sigma_{[H]} / G$, where $\Sigma_{[H]} / G$ is the orbit space of the symmetry strata $\Sigma_{[H]}$ ), and formulate a method to produce such a minimal functional basis of $\Sigma_{[H]}$ (theorem 3.5). Using this tool, we have been able to produce low-cardinality minimal functional bases for the tetragonal, trigonal, transversely isotropic, and cubic strata of Ela, of cardinal at most 6 (whereas a known integrity basis of the full space Ela contains 297 invariants [27]). Finally, theorem 7.1 provides a minimal polynomial functional basis for the elasticity tensors which are at least tetragonal or trigonal, and consists of eight invariants.

\section{APPENDIX A. ElASTICITY SYMMETRY GROUPS}

For each of the eight symmetry classes of the elasticity tensor, as detailed in [17], we provide an explicit representative subgroup $H \subset \mathrm{SO}(3)$ in this class, which serves as a prototype for visualising each of these symmetries.

- $\mathbb{1}$ is the subgroup of $\mathrm{SO}(3)$ reduced to the identity element;

- $\mathbb{Z}_{2}$ is generated by the second-order rotation $\mathbf{r}\left(\boldsymbol{e}_{3}, \pi\right)$. It has order 2 ;

- $\mathbb{D}_{2}$ is generated by the second-order rotations $\mathbf{r}\left(\boldsymbol{e}_{3}, \pi\right)$ and $\mathbf{r}\left(\boldsymbol{e}_{1}, \pi\right)$. It has order 4 ;

- $\mathbb{D}_{3}$ is generated by the third order rotation $\mathbf{r}\left(\boldsymbol{e}_{3}, \frac{2 \pi}{3}\right)$ and the second-order rotation $\mathbf{r}\left(\boldsymbol{e}_{1}, \pi\right)$. It has order 6 ;

- $\mathbb{D}_{4}$ is generated by the fourth-order rotation $\mathbf{r}\left(\boldsymbol{e}_{3}, \frac{\pi}{2}\right)$ and the second-order rotation $\mathbf{r}\left(\boldsymbol{e}_{1}, \pi\right)$. It has order 8;

- $\mathbb{O}$ is the octahedral group, the orientation-preserving symmetry group of the cube with vertices $( \pm 1, \pm 1, \pm 1)$. Its principal directions are the normals to its faces, which are the basis vectors $\pm \boldsymbol{e}_{i}$. It has order 24 ;

- $\mathrm{O}(2)$ is the subgroup generated by all rotations $\mathbf{r}\left(\boldsymbol{e}_{3}, \theta\right)(\theta \in[0 ; 2 \pi[)$ and the second-order rotation $\mathbf{r}\left(\boldsymbol{e}_{1}, \pi\right)$. It is of infinite order.

All these subgroups are compact. The notation $\mathbf{r}(\boldsymbol{n}, \theta)$ denotes a rotation of angle $\theta$ around axis $\langle\boldsymbol{n}\rangle$.

\section{Appendix B. StratificAtion OF FOURTH-ORDER HARMONIC TENSORS}

The vector space $\mathbb{H}^{4}\left(\mathbb{R}^{3}\right)$, of fourth-order harmonic tensors, splits into the following eight symmetry classes (the same as for the elasticity tensor), resulting into the following isotropy stratification of $\mathbb{H}^{4}\left(\mathbb{R}^{3}\right)$ $[20]$

$$
\mathbb{H}^{4}\left(\mathbb{R}^{3}\right)=\Sigma_{[\mathbb{1}]} \cup \Sigma_{\left[\mathbb{Z}_{2}\right]} \cup \Sigma_{\left[\mathbb{D}_{2}\right]} \cup \Sigma_{\left[\mathbb{D}_{3}\right]} \cup \Sigma_{\left[\mathbb{D}_{4}\right]} \cup \Sigma_{[\mathrm{O}(2)]} \cup \Sigma_{[\mathbb{O}]} \cup \Sigma_{[\mathrm{SO}(3)]},
$$

namely into triclinic, monoclinic, orthotropic, trigonal, tetragonal, transversely isotropic, cubic and isotropic strata.

Necessary and sufficient covariant conditions characterizing each symmetry stratum of $\mathbb{H}^{4}\left(\mathbb{R}^{3}\right)$ have been derived in [29, Theorems 9.3, 9.11, 9.15, and Corollary 9.7]. Some of these conditions which are necessary for our purpose are stated below, as theorem B.1 (recall that $\mathbf{d}_{2}$ is transversely isotropic, iff $\mathbf{d}_{2} \times \mathbf{d}_{2}{ }^{2}=0$ and $\mathbf{d}_{2}^{\prime} \neq 0$ ). The conditions for the orthotropic and monoclinic cases require the introduction of additional covariants,

$$
\mathbf{c}_{3}:=\mathbf{H}: \mathbf{d}_{2}=2 \mathbf{d}_{3}^{\prime}, \quad \mathbf{c}_{4}:=\mathbf{H}: \mathbf{c}_{3}, \quad \boldsymbol{v}_{5}:=\varepsilon:\left[\mathbf{d}_{2}, \mathbf{c}_{3}\right], \quad \boldsymbol{v}_{6}:=\varepsilon:\left[\mathbf{d}_{2}, \mathbf{c}_{4}\right],
$$

of order 2 (the $\mathbf{c}_{k}$ ) and order 1 (the $\boldsymbol{v}_{k}$ ).

Theorem B.1. Let $\mathbf{H} \in \mathbb{H}^{4}\left(\mathbb{R}^{3}\right)$ be a fourth-order harmonic tensor. Then

(1) $\mathbf{H}$ is isotropic iff $\mathbf{H}=0$ (i.e. $I_{3}=I_{4}=0$ );

(2) $\mathbf{H}$ is cubic iff $\mathbf{H} \neq 0$ and $\mathbf{d}_{2}$ is isotropic $\left(\mathbf{d}_{2} \neq 0\right.$ and $\left.\mathbf{d}_{2}^{\prime}=0\right)$;

(3) $\mathbf{H}$ is transversely isotropic iff $\mathbf{d}_{2}$ is transversely isotropic and $\mathbf{H} \times \mathbf{d}_{2}=0$;

(4) $\mathbf{H}$ is tetragonal iff $\mathbf{d}_{2}$ is transversely isotropic, $\mathbf{H} \times \mathbf{d}_{2} \neq 0$, and $\operatorname{tr}\left(\mathbf{H} \times \mathbf{d}_{2}\right)=0$;

(5) $\mathbf{H}$ is trigonal iff $\mathbf{d}_{2}$ is transversely isotropic, $\operatorname{tr}\left(\mathbf{H} \times \mathbf{d}_{2}\right) \neq 0$, and $\left(\mathbf{H}: \mathbf{d}_{2}\right) \times \mathbf{d}_{2}=0$;

(6) $\mathbf{H}$ is orthotropic iff $\boldsymbol{v}_{5}=\boldsymbol{v}_{6}=0$ and the pair $\left(\mathbf{d}_{2}, \mathbf{c}_{3}\right)$ is orthotropic;

(7) $\mathbf{H}$ is monoclinic iff the triplet $\left(\mathbf{d}_{2}, \mathbf{c}_{3}, \mathbf{c}_{4}\right)$ is monoclinic.

(8) $\mathbf{H}$ is triclinic iff the triplet $\left(\mathbf{d}_{2}, \mathbf{c}_{3}, \mathbf{c}_{4}\right)$ is triclinic. 
Remark B.2. Polynomial equations involving invariants instead of covariants have been formulated in [3], for some symmetry strata of $\mathbb{H}^{4}\left(\mathbb{R}^{3}\right)$ (those of dimension at most 3 ). They consist in a finite set of polynomial relations and inequalities on the $I_{k}$. For instance, we have $\mathbf{H}=0 \Longleftrightarrow \mathbf{d}_{2}=\mathbf{0} \Longleftrightarrow$ $I_{2}=\|\mathbf{H}\|^{2}=0, \mathbf{d}_{2}^{\prime}=0 \Longleftrightarrow I_{4}=\left\|\mathbf{d}_{2}^{\prime}\right\|^{2}=0$ and, by $(5.13)$, we get that $\operatorname{tr}\left(\mathbf{H} \times \mathbf{d}_{2}\right)=0 \Longleftrightarrow$ $2 I_{2}^{3}-60 I_{3}^{2}-9 I_{4} I_{2}+18 I_{6}=0$. The condition $I_{4}=0$ characterizes the symmetry classes which are at least cubic, and we have $I_{4} \neq 0$ for each fourth-order harmonic tensor which is either transversely isotropic, tetragonal or trigonal.

\section{Appendix C. A ReConstruction formula}

We propose a reconstruction formula for each transversely isotropic tensor $\mathbf{H} \in \Sigma_{[\mathrm{O}(2)]}$ by means of a transversely isotropic second-order tensor t. Denoting by $\langle\boldsymbol{n}\rangle$ (where $\|\boldsymbol{n}\|=1$ ), the axis of transverse isotropy, we introduce $\mathbf{t}:=(\boldsymbol{n} \otimes \boldsymbol{n})^{\prime}$, which belongs to $\mathbb{H}^{2}\left(\mathbb{R}^{3}\right)$. Using the concept of harmonic square introduced in [28], which builds a fourth-order harmonic tensor $\mathbf{t} * \mathbf{t}$ from a second-order harmonic tensor t, we get

$$
\mathbf{t} * \mathbf{t}:=\mathbf{H}(\mathbf{t} \odot \mathbf{t})=\mathbf{t} \odot \mathbf{t}-\frac{4}{7} \mathbf{q} \odot \mathbf{t}^{2}+\frac{2}{35}\|\mathbf{t}\|^{2} \mathbf{q} \odot \mathbf{q} \in \mathbb{H}^{4}\left(\mathbb{R}^{3}\right),
$$

where $\odot$ is the symmetric tensor product and $\mathbf{H}(\mathbf{S})$, defined by $(2.5)$, is the projection of a totally symmetric fourth-order tensor $\mathbf{S}$ onto its fourth-order harmonic component $\mathbf{H}$. It is such that

$$
\|\mathbf{t} * \mathbf{t}\|^{2}=\mathbf{t}:(\mathbf{t} * \mathbf{t}): \mathbf{t}=\frac{8}{35}, \quad(\mathbf{t} * \mathbf{t}):(\mathbf{t} * \mathbf{t})=\frac{8}{105} \mathbf{q}+\frac{12}{147} \mathbf{t} .
$$

We have then a reconstruction formula for $\mathbf{H}$, using the scalar $\mathbf{t}: \mathbf{H}: \mathbf{t}$ and the deviatoric transversely isotropic second-order tensor $\mathbf{t}$ :

Theorem C.1. Each fourth-order harmonic tensor $\mathbf{H} \in \Sigma_{[\mathrm{O}(2)]}$, transversely isotropic of axis $\langle\boldsymbol{n}\rangle$, with $\|\boldsymbol{n}\|=1$, can be written

$$
\mathbf{H}=\frac{35}{8}(\mathbf{t}: \mathbf{H}: \mathbf{t}) \mathbf{t} * \mathbf{t}, \quad \mathbf{t}: \mathbf{H}: \mathbf{t}=\frac{28}{9} \frac{I_{3}}{I_{2}}=\frac{4}{3} \frac{I_{5}}{I_{4}},
$$

with $\mathbf{t}=(\boldsymbol{n} \otimes \boldsymbol{n})^{\prime}$, and

$$
\mathbf{d}_{2}(\mathbf{H})=\frac{5}{48}(\mathbf{t}: \mathbf{H}: \mathbf{t})^{2}(14 \mathbf{q}+15 \mathbf{t})
$$

Proof. It has been shown in [28, Theorem 5.2] that every transversely isotropic fourth-order harmonic tensor $\mathbf{H} \in \mathbb{H}^{4}\left(\mathbb{R}^{3}\right)$ can be reconstructed as

$$
\mathbf{H}=\frac{63}{25 I_{3}} \mathbf{d}_{2}^{\prime} * \mathbf{d}_{2}^{\prime}, \quad I_{3}=\operatorname{tr} \mathbf{d}_{3} .
$$

The evaluations $(5.5)$ of the invariants $I_{k}$ for all $\mathbf{H} \in \Sigma_{[\mathrm{O}(2)]}$ give $63 / 25 I_{3}=35 I_{5} / 9 I_{4}^{2}$ and $28 I_{3} / 9 I_{2}=$ $4 I_{5} / 3 I_{4}$. We have thus

$$
\mathbf{H}=\frac{63}{25 I_{3}} \mathbf{d}_{2}^{\prime} * \mathbf{d}_{2}^{\prime}=\frac{35 I_{5}}{9 I_{4}^{2}}\left(\mathbf{d}_{2}^{\prime} * \mathbf{d}_{2}^{\prime}\right)=\frac{35}{8}(\mathbf{t}: \mathbf{H}: \mathbf{t}) \mathbf{t} * \mathbf{t}
$$

where the last equality results from $\mathbf{t}: \mathbf{H}: \mathbf{t}=4 I_{5} / 3 I_{4}$ (see (6.7)). We get thus (C.2). Finally, since $\mathbf{d}_{2}(\mathbf{H})=\mathbf{H}: \mathbf{H}$, we deduce (C.3) from (C.2) and (C.1).

\section{Appendix D. Separating SETS FOR A PAIR $(\mathbf{H}, \mathbf{t})$}

We provide here separating sets for a pair $(\mathbf{H}, \mathbf{t})$, on the union of strata

$$
\Sigma_{[\mathrm{O}(2)]} \cup \Sigma_{\left[\mathbb{D}_{3}\right]} \cup \Sigma_{\left[\mathbb{D}_{4}\right]}
$$

where $\mathbf{H}$ is a fourth-order harmonic tensor and $\mathbf{t}$ is a transversely isotropic deviator.

Lemma D.1. Let $\mathbf{H} \in \mathbb{H}^{4}\left(\mathbb{R}^{3}\right)$ be a fourth-order harmonic tensor and $\mathbf{t}=(\boldsymbol{n} \otimes \boldsymbol{n})^{\prime}$ with $\|\boldsymbol{n}\|=1$, a deviatoric transversely isotropic tensor. If the pair $(\mathbf{H}, \mathbf{t})$ is at least tetragonal, then all the $I_{k}(\mathbf{H})$ defined by (5.1) are polynomial functions of $I_{2}(\mathbf{H})$ and $\mathbf{t}: \mathbf{H}: \mathbf{t}$. In particular

$$
I_{3}=\frac{3}{4}(\mathbf{t}: \mathbf{H}: \mathbf{t}) I_{2}-\frac{15}{8}(\mathbf{t}: \mathbf{H}: \mathbf{t})^{3}, \quad I_{4}=\frac{1}{6}\left(I_{2}-\frac{15}{2}(\mathbf{t}: \mathbf{H}: \mathbf{t})^{2}\right)^{2} .
$$

The same result holds if the pair $(\mathbf{H}, \mathbf{t})$ is at least trigonal, but with

$$
I_{3}=-\frac{9}{8}(\mathbf{t}: \mathbf{H}: \mathbf{t}) I_{2}+\frac{405}{64}(\mathbf{t}: \mathbf{H}: \mathbf{t})^{3}, \quad I_{4}=\frac{1}{96}\left(I_{2}-\frac{135}{8}(\mathbf{t}: \mathbf{H}: \mathbf{t})^{2}\right)^{2} .
$$


Proof. Suppose first that $(\mathbf{H}, \mathbf{t})$ is at least tetragonal. Without loss of generality, we can assume that its symmetry group contains $\mathbb{D}_{4}$ (defined in Appendix A), and thus that $\mathbf{t}=\left(\boldsymbol{e}_{3} \otimes \boldsymbol{e}_{3}\right)^{\prime}$. Using the Kelvin representation (5.6) of $\mathbf{H}$, we get $\mathbf{t}: \mathbf{H}: \mathbf{t}=H_{3333}=8 \delta$ and (5.7), from which we deduce (D.1). Besides, each invariant $I_{k}(\mathbf{H})$ can be expressed as a polynomial function of $\delta$ and $\sigma^{2}$ by $(5.7)$, and thus of $\mathbf{t}: \mathbf{H}: \mathbf{t}$ and $I_{2}$, which concludes the proof for the tetragonal case. The proof for the trigonal case is similar, except that the Kelvin representation (5.9) leads to $\mathbf{t}: \mathbf{H}: \mathbf{t}=H_{3333}=8 \delta$ and (5.10), and thus to (D.2).

The following theorem is a corollary of lemma D.1 and of result [29, Lemma 8.8], which we recall now.

Lemma D.2. Let $\mathbf{t}$ be a transversely isotropic second-order tensor and $\mathbf{H} \in \mathbb{H}^{4}\left(\mathbb{R}^{3}\right)$. Then, $(\mathbf{H}, \mathbf{t})$ is at least tetragonal if and only if $\operatorname{tr}(\mathbf{H} \times \mathbf{t})=0$.

Theorem D.3. Let $\mathbf{H} \in \mathbb{H}^{4}\left(\mathbb{R}^{3}\right)$ be a fourth-order harmonic tensor and $\mathbf{t}=(\boldsymbol{n} \otimes \boldsymbol{n})^{\prime}$ with $\|\boldsymbol{n}\|=1$, a deviatoric transversely isotropic tensor. Then, the set of invariants

$$
I_{3}:=\operatorname{tr} \mathbf{d}_{3}, \quad I_{4}:=\operatorname{tr} \mathbf{d}_{2}^{\prime 2}=\left\|\mathbf{d}_{2}^{\prime}\right\|^{2}, \quad\|\operatorname{tr}(\mathbf{H} \times \mathbf{t})\|^{2}, \quad \mathbf{t}: \mathbf{H}: \mathbf{t}
$$

is separating for the pair $(\mathbf{H}, \mathbf{t})$ on $\Sigma_{[\mathrm{O}(2)]} \cup \Sigma_{\left[\mathbb{D}_{3}\right]} \cup \Sigma_{\left[\mathbb{D}_{4}\right]}$.

Proof. Let $(\mathbf{H}, \mathbf{t})$ and $(\overline{\mathbf{H}}, \overline{\mathbf{t}})$ in $\Sigma_{[\mathrm{O}(2)]} \cup \Sigma_{\left[\mathbb{D}_{3}\right]} \cup \Sigma_{\left[\mathbb{D}_{4}\right]}$, with $\mathbf{t}=(\boldsymbol{n} \otimes \boldsymbol{n})^{\prime}$ and $\overline{\mathbf{t}}=(\overline{\boldsymbol{n}} \otimes \overline{\boldsymbol{n}})^{\prime}$, and assume that the four invariants (D.3) have the same values on $(\mathbf{H}, \mathbf{t})$ and $(\overline{\mathbf{H}}, \overline{\mathbf{t}})$. Suppose first $\operatorname{that} \operatorname{tr}(\overline{\mathbf{H}} \times \overline{\mathbf{t}})=$ $\operatorname{tr}(\mathbf{H} \times \mathbf{t})=0$, then, by lemma D.2, we conclude that both $(\mathbf{H}, \mathbf{t})$ and $(\overline{\mathbf{H}}, \overline{\mathbf{t}})$ are at least tetragonal. Then, there exists a rotation $g$ such that $g \star \boldsymbol{n}=\boldsymbol{e}_{3}$ and $[g \star \mathbf{H}]$ can be written as (5.6) with parameters $(\delta, \sigma)$. Similarly, there exists a rotation $\bar{g}$ such that $\bar{g} \star \overline{\boldsymbol{n}}=\boldsymbol{e}_{3}$ and $[\bar{g} \star \overline{\mathbf{H}}]$ can be written as (5.6) with parameters $(\bar{\delta}, \bar{\sigma})$. Now, by lemma D.1, we deduce that, in any case we have $(\bar{\delta}, \bar{\sigma})=(\delta, \pm \sigma)$ and thus that either $(\overline{\mathbf{H}}, \overline{\mathbf{t}})=\left(\bar{g}^{-1} g\right) \star(\mathbf{H}, \mathbf{t})$ or $(\overline{\mathbf{H}}, \overline{\mathbf{t}})=\left(\bar{g}^{-1} r g\right) \star(\mathbf{H}, \mathbf{t})$, where $r=\mathbf{r}\left(\boldsymbol{e}_{3}, \frac{\pi}{4}\right)$ is the rotation of angle $\pi / 4$ about $\boldsymbol{e}_{3}$. If $\operatorname{tr}(\overline{\mathbf{H}} \times \overline{\mathbf{t}})=\operatorname{tr}(\mathbf{H} \times \mathbf{t}) \neq 0$, then, $(\mathbf{H}, \mathbf{t})$ and $(\overline{\mathbf{H}}, \overline{\mathbf{t}})$ are both at least trigonal, and the arguments are similar.

\section{Appendix E. Proof of TheOREM 7.1}

Observe first that an elasticity tensor $\mathbf{E} \in \mathbf{X}$ is at least cubic if and only if

$$
K_{4}:=\left\|\mathbf{d}^{\prime}\right\|^{4}+\left\|\mathbf{v}^{\prime}\right\|^{4}+\left\|\mathbf{d}_{2}^{\prime}\right\|^{2}=0 .
$$

If $K_{4} \neq 0$, then, $\mathbf{E}=\left(\operatorname{tr} \mathbf{d}, \operatorname{tr} \mathbf{v}, \mathbf{d}^{\prime}, \mathbf{v}^{\prime}, \mathbf{H}\right)$ belongs thus to $\Sigma_{\left[\mathbb{D}_{3}\right]} \cup \Sigma_{\left[\mathbb{D}_{4}\right]} \cup \Sigma_{[\mathrm{O}(2)]}$, the transversely isotropic rational covariant $\mathbf{t}:=2 \mathbf{k}_{4} / K_{4}$ is well defined and by (6.4), and we have

$$
\mathbf{d}^{\prime}=\frac{3 K_{5}}{K_{4}} \mathbf{t}, \quad \mathbf{v}^{\prime}=\frac{3 L_{5}}{K_{4}} \mathbf{t}, \quad \mathbf{d}_{2}^{\prime}= \pm \sqrt{\frac{3}{2}}\left\|\mathbf{d}_{2}^{\prime}\right\| \mathbf{t} .
$$

Moreover, $\mathbf{E}$ has the same symmetry class as the pair $(\mathbf{H}, \mathbf{t})$ which is either trigonal, tetragonal or transversely isotropic.

By theorems 6.1 to 6.5 , the set $\mathscr{F}:=\left\{K_{1}, L_{1}, \ldots, K_{10}\right\}$ is separating for each individual stratum $\Sigma_{[H]}$ contained in $\mathbf{X}$. Therefore, given two elasticity tensors $\mathbf{E}, \overline{\mathbf{E}} \in \mathbf{X}$ with the same eight invariants $K_{1}=\bar{K}_{1}, L_{1}=\bar{L}_{1}, \ldots, K_{10}=\bar{K}_{10}$, to prove that they are in the same orbit it is enough to show that they belong to the same symmetry class. Therefore, let $\overline{\mathbf{E}}=\left(\operatorname{tr} \overline{\mathbf{d}}, \operatorname{tr} \overline{\mathbf{v}}, \overline{\mathbf{d}^{\prime}}, \overline{\mathbf{v}^{\prime}}, \overline{\mathbf{H}}\right)$, and we will argue according to the symmetry class of $\mathbf{E}$.

(A) If $\mathbf{E}$ is isotropic, then, all invariants in $\mathscr{F} \backslash\left\{K_{1}, L_{1}\right\}$ vanish. Hence,

$$
K_{4}=\bar{K}_{4}=\left\|\overline{\mathbf{d}^{\prime}}\right\|^{4}+\left\|\overline{\mathbf{v}^{\prime}}\right\|^{4}+\left\|\overline{\mathbf{d}_{2}^{\prime}}\right\|^{2}=0 \Longrightarrow \overline{\mathbf{d}^{\prime}}=\overline{\mathbf{v}^{\prime}}=\overline{\mathbf{d}_{2}^{\prime}}=\mathbf{0} .
$$

Therefore, by theorem B.1, $\overline{\mathbf{H}}$ is at least cubic, and since $I_{3}=\bar{I}_{3}=0$, we conclude by (5.3), that $\overline{\mathbf{H}}=0$, and thus that $\overline{\mathbf{E}}$ is isotropic.

(B) If $\mathbf{E}$ is cubic, then, all invariants in $\mathscr{F} \backslash\left\{K_{1}, L_{1}, I_{3}\right\}$ vanish but $I_{3}=\bar{I}_{3} \neq 0$. We conclude, as in case (A), that $\overline{\mathbf{E}}$ is at least cubic, and indeed cubic, since $\bar{I}_{3} \neq 0$ and thus $\bar{I}_{2}=\|\overline{\mathbf{H}}\|^{2} \neq 0$.

(C) If $\mathbf{E}$ is either transversely isotropic, trigonal or tetragonal, then, $\overline{K_{4}}=K_{4} \neq 0$ and thus $\overline{\mathbf{E}}$ is either transversely isotropic, trigonal or tetragonal. Hence, $\overline{\mathbf{t}}$ is well-defined and is written

$$
\overline{\mathbf{t}}:=\frac{2}{\bar{K}_{4}}\left(\left\|{\overline{\mathbf{d}^{\prime}}}^{2}{\overline{\mathbf{d}^{\prime}}}^{2}+\right\|{\overline{\mathbf{v}^{\prime}}}^{2}{\overline{\mathbf{v}^{\prime}}}^{2}+{\overline{\mathbf{d}_{2}^{\prime}}}^{2}\right)^{\prime},
$$


and $\overline{\mathbf{E}}$ has the same symmetry class as the pair $(\overline{\mathbf{H}}, \overline{\mathbf{t}})$. Now, from (E.1) and since $\|\mathbf{t}\|^{2}=\|\overline{\mathbf{t}}\|^{2}=2 / 3$, $K_{5}=\bar{K}_{5}, L_{5}=\bar{L}_{5}$,

$$
K_{4}=\frac{36}{K_{4}^{4}}\left(K_{5}^{4}+L_{5}^{4}\right)+\left\|\mathbf{d}_{2}^{\prime}\right\|^{2}=\bar{K}_{4}=\frac{36}{\bar{K}_{4}^{4}}\left(\bar{K}_{5}^{4}+\bar{L}_{5}^{4}\right)+\left\|\overline{\mathbf{d}_{2}^{\prime}}\right\|^{2},
$$

we get $\bar{I}_{4}=\left\|\overline{\mathbf{d}_{2}^{\prime}}\right\|^{2}=\left\|\mathbf{d}_{2}^{\prime}\right\|^{2}=I_{4}$. Therefore, by theorem D.3, $(\mathbf{H}, \mathbf{t})$ and $(\overline{\mathbf{H}}, \overline{\mathbf{t}})$ are in the same orbit, and belong thus to the same symmetry class.

So far, we have proved that the family $\mathscr{F}=\left\{K_{1}, L_{1}, \ldots, K_{10}\right\}$ is separating for the three cases (1), (2) and (3) of theorem 7.1. Note moreover, that $K_{10}$ can be removed from $\mathscr{F}$ when the goal is to separate "at least tetragonal" (case (1)) or "at least trigonal" (case (2)) elasticity tensors. Indeed, in the proof of theorem D.3, $K_{10}$ is used only to distinguish whether a pair $(\mathbf{H}, \mathbf{t})$ is tetragonal or trigonal.

Finally, it remains to prove the minimality of the separating set $\mathscr{F}$ for X, and of $\mathscr{F} \backslash\left\{K_{10}\right\}$ for cases (1) and (2). Let $\mathscr{F}^{\prime}$ be a proper subset of $\mathscr{F}$ or of $\mathscr{F} \backslash\left\{K_{10}\right\}$.

(a) If $\mathscr{F}^{\prime}$ does not contain $K_{1}=\operatorname{tr} \mathbf{d}$ or $L_{1}=\operatorname{tr} \mathbf{v}$, then it fails to be a separating set for isotropic elasticity tensors.

(b) If $\mathscr{F}^{\prime}$ does not contain $I_{3}=\operatorname{tr} \mathbf{d}_{3}$, then it fails to be a separating set for cubic elasticity tensors.

(c) If $\mathscr{F}^{\prime}$ does not contain $K_{5}=\mathbf{d}: \mathbf{k}_{4}$, then the two transversely isotropic elasticity tensors $\mathbf{E}_{1}=$ $\left(0,0, \mathbf{d}^{\prime}, 0,0\right)$ and $\mathbf{E}_{2}=\left(0,0,-\mathbf{d}^{\prime}, 0,0\right)$ have the same values for $\mathscr{F}^{\prime}$ but are not on the same orbit. The same conclusion holds for $L_{5}=\mathbf{v}: \mathbf{k}_{4}$.

(d) If $\mathscr{F}^{\prime}$ does not contain $K_{4}=\left\|\mathbf{d}^{\prime}\right\|^{4}+\left\|\mathbf{v}^{\prime}\right\|^{4}+I_{4}$, then it fails to separate an harmonic tetragonal tensor $\mathbf{H}_{\mathbb{D}_{4}}$ with $\delta=0$ (see (5.6)) from $\mathbf{E}=0$, since all the invariants in $\mathscr{F}^{\prime}$ vanish on these tensors.

(e) If $\mathscr{F}^{\prime}$ does not contain $K_{9}=\mathbf{k}_{4}: \mathbf{H}: \mathbf{k}_{4}$, it fails to separate tetragonal harmonic tensors (by remark 5.4) and to separate trigonal harmonic tensors (by remark 5.6).

(f) Finally, if $\mathscr{F}^{\prime}$ does not contain $K_{10}=\left\|\operatorname{tr}\left(\mathbf{H} \times \mathbf{k}_{4}\right)\right\|^{2}$, then it fails to be a separating set for $\mathbf{X}$ since all the invariants in $\mathscr{F}^{\prime}$ take the same values on the harmonic trigonal tensor $\mathbf{H}_{\mathbb{D}_{3}}$ with $\delta=0$ and $\sigma=\sigma_{1} \neq 0\left(\right.$ see (5.9)) and the harmonic tetragonal tensor $\mathbf{H}_{\mathbb{D}_{4}}$ with $\delta=0$ and $\sigma=\sigma_{2}$ (see $\left.(5.6)\right)$, when $\sigma_{2}^{2}=2 \sigma_{1}^{2}$.

These arguments show that $\mathscr{F}$ is a minimal separating set for $\mathrm{X}$, which proves point (3) of theorem 7.1. Items (a) to (e) show that $\mathscr{F} \backslash\left\{K_{10}\right\}$ is a minimal separating set for either at least tetragonal tensors (point (1)) or at least trigonal tensors (point (2)), which achieves the proof.

Funding. The authors were partially supported by CNRS Projet 80-Prime GAMM (Géométrie algébrique complexe/réelle et mécanique des matériaux).

\section{REFERENCES}

[1] S. Abramian, B. Desmorat, R. Desmorat, B. Kolev, and M. Olive. Recovering the normal form and symmetry class of an elasticity tensor. J. Elasticity, 142(1):1-33, jul 2020.

[2] M. Abud and G. Sartori. The geometry of spontaneous symmetry breaking. Ann. Physics, 150(2):307-372, 1983.

[3] N. Auffray, B. Kolev, and M. Petitot. On Anisotropic Polynomial Relations for the Elasticity Tensor. J. Elasticity, 115(1):77-103, June 2014

[4] G. Backus. A geometrical picture of anisotropic elastic tensors. Rev. Geophys., 8(3):633-671, 1970.

[5] R. Baerheim. Harmonic decomposition of the anisotropic elasticity tensor. Q. J. Mech. Appl. Math., 46(3):391-418, 1993.

[6] J.-P. Boehler. Introduction to the invariant formulation of anisotropic constitutive equations. In Applications of tensor functions in solid mechanics, volume 292 of CISM Courses and Lectures, pages 13-30. Springer, Vienna, 1987.

[7] J.-P. Boehler, A. A. Kirillov, Jr., and E. T. Onat. On the polynomial invariants of the elasticity tensor. J. Elasticity, 34(2):97-110, 1994.

[8] A. Bóna, I. Bucataru, and M. A. Slawinski. Space of SO(3)-orbits of elasticity tensors. Arch. Mech. (Arch. Mech. Stos.), 60(2):123-138, 2008.

[9] G. E. Bredon. Finiteness of number of orbit types. In A. Borel, Seminar on transformation groups. With contributions by G. Bredon, EE Floyd, D. Montgomery, R. Palais. Annals of Mathematics Studies, number 46, 1960.

[10] G. E. Bredon. Introduction to compact transformation groups. Academic Press, New York, 1972. Pure and Applied Mathematics, Vol. 46.

[11] G. E. Bredon. Topology and geometry. Graduate Texts in Mathematics. Springer, corrected edition, 1993.

[12] L. Brouwer. Beweis der invarianz des n-dimensionalen gebiets. Math. Ann., 71:305-313, 1912.

[13] Z. Chen, J. Liu, L. Qi, Q. Zheng, and W. Zou. An irreducible function basis of isotropic invariants of a third order three-dimensional symmetric tensor. J. Math. Phys., 59:081703, 2018.

[14] S. C. Cowin. Properties of the anisotropic elasticity tensor. Q. J. Mech. Appl. Math., 42:249-266, 1989.

[15] R. Desmorat, N. Auffray, B. Desmorat, B. Kolev, and M. Olive. Generic separating sets for three-dimensional elasticity tensors. Proc. R. Soc. A, 475, 2019.

[16] J. Draisma, G. Kemper, and D. Wehlau. Polarization of separating invariants. Canad. J. Math., 60(3):556-571, 2008.

[17] S. Forte and M. Vianello. Symmetry classes for elasticity tensors. J. Elasticity, 43(2):81-108, 1996. 
[18] D. Hilbert. Theory of algebraic invariants. Cambridge University Press, Cambridge, 1993.

[19] J. G. Hocking and G. S. Young. Topology. Dover Publications Inc., New York, 1988.

[20] E. Ihrig and M. Golubitsky. Pattern selection with $\mathrm{O}(3)$ symmetry. 13(1-2):1-33, 1984.

[21] H. Kraft and C. Procesi. Classical Invariant Theory, a Primer. Lectures notes avaiable at http://www.math.unibas . $\mathrm{ch} / \sim \mathrm{kraft} /$ Papers/KP-Primer.pdf, 2000.

[22] J. J. Liu, W. Y. Ding, L. Q. Qi, and W. N. Zou. Isotropic polynomial invariants of the hall tensor. Appl. Math. Mech.-Engl. Ed., 39(12):1845-1856, 2018.

[23] Z. Ming, Y. Chen, L. Qi, and L. Zhang. A polynomially irreducible functional basis of elasticity tensors. arXiv preprint arXiv:1912.03077, 2019.

[24] Z. Ming, L. Zhang, and Y. Chen. An irreducible polynomial functional basis of two-dimensional eshelby tensors. Appl. Math. Mech.-Engl. Ed., 40(8):1169-1180, 2019.

[25] M. Olive. About Gordan's algorithm for binary forms. Found. Comput. Math., 17(6):1407-1466, 2017.

[26] M. Olive and N. Auffray. Isotropic invariants of a completely symmetric third-order tensor. Journal of Mathematical Physics, 55(9):092901, 2014.

[27] M. Olive, B. Kolev, and N. Auffray. A minimal integrity basis for the elasticity tensor. Arch. Ration. Mech. Anal., 226(1):1-31, Oct. 2017.

[28] M. Olive, B. Kolev, R. Desmorat, and B. Desmorat. Harmonic factorization and reconstruction of the elasticity tensor. Journal of Elasticity, 101:132-67, 2018.

[29] M. Olive, B. Kolev, R. Desmorat, and B. Desmorat. Characterization of the symmetry class of an elasticity tensor using polynomial covariants. Mathematics and Mechanics of Solids, may 2021.

[30] P. J. Olver. Equivalence, invariants, and symmetry, volume 44. Cambridge University Press, Cambridge, 1995.

[31] A. Pipkin and R. S. Rivlin. The formulation of constitutive equations in continuum physics I. Arch. Rational Mech. Anal., 4:129-144 (1959), 1959.

[32] A. Pipkin and A. Wineman. Material symmetry restrictions on non-polynomial constitutive equations. Arch. Rational Mech. Anal., 12:420-426, 1963.

[33] J. Rychlewski. On hooke's law. Prikl. Matem. Mekhan., 48:303-314, 1984

[34] T. Shioda. On the graded ring of invariants of binary octavics. Amer. J. Math., 89:1022-1046, 1967.

[35] G. Smith. On isotropic functions of symmetric tensors, skew-symmetric tensors and vectors. Int. J. Eng. Sci., 9:899-916, 1971.

[36] B. Sturmfels. Algorithms in Invariant Theory. Texts \& Monographs in Symbolic Computation. $2^{\text {nd }}$ edition, Springer Wien New-York, 2008.

[37] W. K. Thomson (Lord Kelvin). Elasticity, Encyclopaedia Britannica. Adam and Charles Black, Edinburgh, 1878.

[38] P. Vannucci and G. Verchery. Stiffness design of laminates using the polar method. International Journal of Solids and Structures, 38:9281-9894, 2001.

[39] G. Verchery. Les invariants des tenseurs d'ordre 4 du type de l'élasticité. In J.-P. Boehler, editor, Colloque Int. CNRS 295, Villard de Lans, pages 93-104. Martinus Nijhoff Publishers and Editions du CNRS, $1982,1979$.

[40] M. Vianello. An integrity basis for plane elasticity tensors. Arch. Mech., 49:197-208, 1997.

[41] C.-C. Wang. Corrigendum to my recent papers on Representations for isotropic functions. Arch. Rational Mech. Anal., 43:392-395, 1970

[42] H. Weyl. The classical groups. Princeton Landmarks in Mathematics. Princeton University Press, Princeton, NJ, 1997. Their invariants and representations, Fifteenth printing, Princeton Paperbacks.

[43] A. Wineman and A. Pipkin. Material symmetry restrictions on constitutive equations. Arch. Ration. Mech. Anal., $17: 184-214,1964$

[44] Q.-S. Zheng. Theory of representations for tensor functions - A unified invariant approach to constitutive equations. Appl. Mech. Rev., 47:545-587, 1994.

Université Paris-Saclay, ENS Paris-Saclay, CNRS, LMT - Laboratoire de Mécanique et Technologie, 91190,

GiF-SUR-Yvette, France

Email address: rodrigue.desmorat@ens-paris-saclay.fr

MSme, Université Paris-Est, Laboratoire Modélisation et Simulation Multi Echelle, MSME UMR 8208

CNRS, 5 Bd Descartes, 77454 Marne-la-Vallée, France

Email address: Nicolas.auffray@univ-mlv.fr

Sorbonne Université, UMPC Univ Paris 06, CNRS, UMR 7190, Institut d'Alembert, F-75252 Paris Cedex 05,

France \& Univ Paris Sud 11, F-91405 Orsay, France

Email address: boris.desmorat@upmc.fr

Université Paris-Saclay, ENS Paris-Saclay, CNRS, LMT - Laboratoire de Mécanique et Technologie, 91190, Gif-Sur-Yvette, France

Email address: marc.olive@math.cnrs.fr

Université Paris-Saclay, ENS Paris-Saclay, CNRS, LMT - Laboratoire de Mécanique et Technologie, 91190, GiF-SUR-Yvette, France

Email address: boris.kolev@math.cnrs.fr 